\title{
Quantum aspects of the higher-derivative Lorentz-breaking extension of QED
}

\author{
T. Mariz, ${ }^{1, *}$ J. R. Nascimento, ${ }^{2, \dagger}$ A. Yu. Petrov, ${ }^{2, *}$ and C. Marat Reyes ${ }^{3, \S}$ \\ ${ }^{1}$ Instituto de Física, Universidade Federal de Alagoas, 57072-270, Maceió, Alagoas, Brazil \\ ${ }^{2}$ Departamento de Física, Universidade Federal da Paraíba, \\ Caixa Postal 5008, 58051-970, João Pessoa, Paraíba, Brazil \\ ${ }^{3}$ Departamento de Ciencias Básicas, Universidad del Bío Bío, Casilla 447, Chillán, Chile
}

(Received 25 April 2018; revised manuscript received 25 March 2019; published 13 May 2019)

\begin{abstract}
We consider the higher-derivative Lorentz-breaking extension of QED, where the new terms are the Myers-Pospelov-like ones in gauge and spinor sectors, and the higher-derivative Carroll-Field-Jackiw term. For this theory, we study its tree-level dynamics, discuss the dispersion relation, and present one more scheme for its perturbative generation, including the finite-temperature case. Also, we develop a method to study perturbative unitarity based on consistent rotation of the theory to Euclidean space. We use this method to verify explicitly that for special choices of the Lorentz-breaking vector the unitarity is preserved at the one-loop level, even in the presence of higher time derivatives.
\end{abstract}

DOI: 10.1103/PhysRevD.99.096012

\section{INTRODUCTION}

Formulation of the Lorentz-breaking extension of the standard model called attention to studies of Lorentzbreaking extensions for many field theory models and, first of all, for QED [1]. Conclusions obtained by treating different aspects of various extensions of QED in dozens of papers became paradigmatic results for Lorentz-breaking theories in general. Among the most important directions of their study, one can emphasize searches of exact solutions, canonical quantization, and calculations of quantum corrections. These studies have allowed us to put strong bounds on Lorentz-violating quantum field theory models [2]. Within this context, an important role is naturally played by higher-derivative Lorentz-breaking extensions of QED. Indeed, it is well known that an effective action is nonlocal and can be represented in the form of the derivative expansion. Moreover, the higher-derivative terms naturally emerge within the string context [3]. Therefore, one naturally faces a problem of studying different issues related to higher-derivative Lorentz-breaking extensions of QED. The first step in such a study has been carried out in Ref. [4], in which the so-called Myers-Pospelov (MP) term, that is,

\footnotetext{
tmariz@fis.ufal.br

jroberto@fisica.ufpb.br

petrov@fisica.ufpb.br

creyes@ubiobio.cl
}

Published by the American Physical Society under the terms of the Creative Commons Attribution 4.0 International license. Further distribution of this work must maintain attribution to the author(s) and the published article's title, journal citation, and DOI. Funded by SCOAP. the first higher-derivative Lorentz-breaking term in QED, has been proposed. This term has attracted great interest due to the fact that a special choice of the Lorentz-breaking vector allows us to rule out the higher time derivatives from this term, thus avoiding unitarity breaking, which is known to be the main problem of higher-derivative theories. A number of studies of unitarity issues for QED with the additive MP term have been performed in Ref. [5]. Some other tree-level results for this theory can be found in Ref. [6], and its phenomenological applications can be found in Ref. [7]. Further, the higher-derivative terms were shown to arise as quantum corrections, first for the case in which the Lorentz symmetry breaking is introduced through the third-rank constant tensor [8] (which for a certain choice of this tensor yields the higher-derivative Carroll-FieldJackiw (CFJ)-like term discussed in Refs. $[9,10]$ ) and, second, for the case in which the Lorentz symmetry breaking is introduced through a constant vector, with the nonminimal coupling present [11]. It was shown that in these cases the resulting higher-derivative terms are finite. Therefore, one can naturally establish the questions, first about other possible schemes allowing us to generate the higher-derivative Lorentz-breaking terms and, second, about the tree-level behavior of the QED with additive higher-derivative Lorentz-breaking terms, which clearly would modify propagators and ultraviolet behavior of the theory. In this paper, we address namely these questions. To be more precise, in this paper, we introduce the higherderivative terms in the gauge sector and discuss the impacts of higher derivatives for the propagator and unitarity.

The structure of this paper is as follows. In Sec. II, we introduce the classical action of the gauge sector of the 
Lorentz-breaking extended QED with higher derivatives. In Sec. III, we carry out the one-loop calculation of the higherderivative Lorentz-breaking terms in the gauge sector with the use of the new coupling, both at zero and finite temperatures. In Sec. IV, we discuss the related unitarity issues and explicitly demonstrate that, even in the presence of the higher time derivatives, unitarity is preserved. Finally, in Sec. V, we summarize our results.

\section{CLASSICAL ACTION AND DISPERSION RELATIONS}

Let us consider the higher-derivative (HD) extension of QED looking like

$\mathcal{L}_{\mathrm{HD}}=-\frac{1}{4} F_{\mu \nu} F^{\mu \nu}-\frac{1}{M} \epsilon^{\beta \mu \nu \lambda} u_{\beta} A_{\mu}\left(c_{1}(u \cdot \partial)^{2}-c_{2} u^{2} \square\right) F_{\nu \lambda}$.

Here, $u_{\mu}$ is a dimensionless vector; $M$ is a mass scale, which is typically suggested to be of the order of the Planck mass [4]; and $c_{1}$ and $c_{2}$ are some dimensionless numbers. The number $c_{1}$ accompanies the Myers-Pospelov term [4], and the number $c_{2}$ accompanies the higher-derivative $\mathrm{CFJ}$ term [10]. We note that within many schemes of calculating the loop corrections both these terms arise, see, for example, Ref. [11]. We note that both these terms are CPT odd, and they represent themselves as specific particular examples of higher-derivative Lorentz-breaking extensions of the gauge sector discussed in details in Ref. [12].

Since this theory is gauge invariant, we can impose the usual Feynman gauge, which does not affect the higherderivative terms. The resulting quadratic Lagrangian for the essentially transversal $A_{\mu}$ will be given by the expression

$$
\mathcal{L}=\frac{1}{2} A_{\mu} \Delta^{\mu \nu} A_{\nu}
$$

with

$$
\Delta^{\mu \lambda}=\square \eta^{\mu \lambda}+\frac{4}{M} \Sigma \epsilon^{\beta \mu \nu \lambda} u_{\beta} \partial_{\nu},
$$

where we introduced the notation $\Sigma=c_{2} u^{2} \square-c_{1}(u \cdot \partial)^{2}$. As a result, one will have just the propagator, the explicit form of which is

$$
\begin{aligned}
G_{\nu \lambda}\left(x-x^{\prime}\right)= & {\left[A_{1} \eta_{\nu \lambda}+A_{2} u_{\nu} u_{\lambda}+A_{3} u_{\nu} \partial_{\lambda}+A_{4} u_{\lambda} \partial_{\nu}\right.} \\
& \left.+A_{5} \partial_{\nu} \partial_{\lambda}+A_{6} \epsilon_{\nu \lambda \rho \sigma} u^{\sigma} \partial^{\rho}\right] \delta^{4}\left(x-x^{\prime}\right) .
\end{aligned}
$$

Defining

$$
D=u^{2} \square-(u \cdot \partial)^{2}, \quad Q=\square^{2}-\frac{16 \Sigma^{2}}{M^{2}} D,
$$

we get

$$
\begin{aligned}
& A_{1}=\frac{\square}{Q}, \quad A_{2}=-\frac{16 \Sigma^{2}}{M^{2} Q}, \\
& A_{3}=A_{4}=\frac{16 \Sigma^{2}(u \cdot \partial)}{M^{2} Q \square}, \\
& A_{5}=\frac{4 \Sigma A_{6} u^{2}}{M \square}=-\frac{16 \Sigma^{2} u^{2}}{M^{2} Q \square}, \quad A_{6}=-\frac{4 \Sigma}{M Q} .
\end{aligned}
$$

Throughout this paper, we are using the definition of the Levi-Civita tensor $\epsilon^{0123}=-\epsilon_{0123}=1$.

In momentum space, we write

$$
\begin{aligned}
G_{\nu \lambda}(p)= & \frac{1}{Q(p)}\left[-p^{2} \eta_{\nu \lambda}-a^{2}\left(u_{\nu} u_{\lambda}-\frac{(u \cdot p)}{p^{2}}\left(u_{\nu} p_{\lambda}+u_{\lambda} p_{\nu}\right)\right.\right. \\
& \left.\left.+\frac{u^{2}}{p^{2}} p_{\nu} p_{\lambda}\right)+a i \epsilon_{\nu \lambda \rho \sigma} u^{\sigma} p^{\rho}\right],
\end{aligned}
$$

where

$$
a=\frac{4 \Sigma(p)}{M}
$$

and $Q(p)$ and $\Sigma(p)$ are just the momentum space counterparts of the same expressions. That is,

$$
Q(p)=\left(p^{2}\right)^{2}-a^{2} D(p),
$$

with

$$
D(p)=(u \cdot p)^{2}-u^{2} p^{2} .
$$

We note that this propagator involves the contributions asymptotically behaving like $\frac{1}{\square}$, which indicates that the UV behavior is the same as in usual theories without higher derivatives (for example, the term $A_{1} \eta_{\nu \lambda}$ asymptotically behaves as $\frac{1}{k^{2}}$, and renormalization properties will not be improved compared with the usual QED. The similar situation occurs in three-dimensional QED with higherderivative CFJ term $\kappa \epsilon^{\mu \nu \lambda} A_{\mu} \partial_{\nu} \square A_{\lambda}$, where one has

$$
\begin{aligned}
{\left[\square\left(\eta^{\mu \nu}+\kappa \epsilon^{\mu \nu \lambda} \partial_{\lambda}\right)\right]^{-1}=} & \frac{\eta_{\nu \rho}}{\square\left(1+\kappa^{2} \square\right)}+\frac{\kappa^{2} \partial_{\nu} \partial_{\rho}}{\square\left(1+\kappa^{2} \square\right)} \\
& -\frac{\kappa \epsilon_{\nu \rho \sigma} \partial^{\sigma}}{\square\left(1+\kappa^{2} \square\right)} .
\end{aligned}
$$

Here, the term proportional to $\partial_{\nu} \partial_{\rho}$ asymptotically behaves as $k^{-2}$; thus, the UV asymptotics is the same as in the usual case.

To find the dispersion relations, one should consider the denominators of (6) and carry out the Fourier transform, so from the denominator $Q$, one finds the unusual dispersion relation (in which $u^{2}=u_{0}^{2}-\vec{u}^{2}$ is the usual square of the vector $u^{\mu}$ in Minkowski space), some aspects of which have been earlier studied also in Ref. [13]: 


$$
\begin{aligned}
& \left(E^{2}-\vec{p}^{2}\right)^{2}+\frac{16}{M^{2}}\left(c_{2} u^{2}\left(E^{2}-\vec{p}^{2}\right)\right. \\
& \left.\quad-c_{1}\left(u_{0} E-\vec{u} \cdot \vec{p}\right)^{2}\right)^{2}\left(u^{2}\left(E^{2}-\vec{p}^{2}\right)\right. \\
& \left.\quad-\left(u_{0} E-\vec{u} \cdot \vec{p}\right)^{2}\right)=0 .
\end{aligned}
$$

This dispersion relation in general cannot be simplified since there is no fundamental reason to impose the relation $c_{1}=c_{2}$ forever. Here, we emphasize some typical situations:

(1) The vector $u^{\mu}$ is lightlike, $u_{\mu} u^{\mu}=0$. In this case, the CFJ-like term vanishes (the same situation is observed if $c_{2}=0$ ), and we have the simplified dispersion relation:

$$
\left(E^{2}-\vec{p}^{2}\right)^{2}-\frac{16 c_{1}^{2}}{M^{2}}\left(u_{0} E-\vec{u} \cdot \vec{p}\right)^{6}=0 .
$$

(2) For $c_{1}=c_{2}$, we have the following simplification of the dispersion relation:

$$
\begin{gathered}
\left(E^{2}-\vec{p}^{2}\right)^{2}+\frac{16 c_{1}^{2}}{M^{2}}\left(u^{2}\left(E^{2}-\vec{p}^{2}\right)\right. \\
\left.-\left(u_{0} E-\vec{u} \cdot \vec{p}\right)^{2}\right)^{3}=0 .
\end{gathered}
$$

(3) The vector $u^{\mu}$ is spacelike, with $u_{0}=0$ and $c_{2}=0$ (no CFJ-like term). In this case, we can avoid the presence of higher time derivatives (so, the theory does not involve ghosts, being hence most likely unitary), and

$$
\begin{aligned}
& \left(E^{2}-\vec{p}^{2}\right)^{2}-\frac{16 c_{1}^{2}}{M^{2}}(\vec{u} \cdot \vec{p})^{4}\left(\vec{u}^{2}\left(E^{2}-\vec{p}^{2}\right)\right. \\
& \left.+(\vec{u} \cdot \vec{p})^{2}\right)=0 .
\end{aligned}
$$

(4) If $u^{\mu}$ is timelike and has only a $u_{0}$ nonzero component, with $u_{i}=0$, we also have the absence of higher time derivatives (so, unitarity is again most likely achieved).

To study unitarity in our theory, we must determine the physical degrees of freedom (d.o.f.) of the gauge field and the correct $i \epsilon$ prescription in order to perform a consistent Wick rotation to Euclidean space, as we explain in Sec. IV.

Let us begin to study the extra conditions on the gauge field, arising through contracting $\partial_{\mu}$ and $u_{\mu}$ with $\Delta^{\mu \lambda}$ in Eq. (3). We obtain $(\partial \cdot A)=(u \cdot A)=0$, which indicates that we must express the gauge field in terms of polarization vectors perpendicular to $p$ and $u$. The strategy to obtain these polarization vectors is to start with two real transverse vectors $e_{\mu}^{(a)}$, with $a=1,2$, and then change to transverse complex ones $\varepsilon_{\mu}^{(\lambda)}$, with $\lambda= \pm$.

Let us consider two linear polarization vectors $e_{\mu}^{(a)}$, satisfying the relation

$$
e_{\mu \nu}=-\sum_{a=1,2} e_{\mu}^{(a)} e_{\nu}^{(b)}
$$

and

$$
\eta^{\mu \nu} e_{\mu}^{(a)} e_{\nu}^{(b)}=-\delta^{a b}
$$

Now, we introduce the projector $P_{\mu \nu}^{(\lambda)}$,

$$
P_{\mu \nu}^{(\lambda)}=\frac{1}{2}\left(e_{\mu \nu}+i \lambda \epsilon_{\mu \nu}\right)
$$

which projects any 4-vector $v^{\mu}$ onto the hyperplane orthogonal to $u^{\nu}$ and $p^{\lambda}$ vectors, with

$e^{\mu \nu}=\eta^{\mu \nu}-\frac{(u \cdot p)}{D}\left(u^{\mu} p^{\nu}+u^{\nu} p^{\mu}\right)+\frac{p^{2}}{D} u^{\mu} u^{\nu}+\frac{u^{2}}{D} p^{\mu} p^{\nu}$,

$$
\epsilon^{\mu \nu}=\frac{\epsilon^{\mu \lambda \rho \nu} u_{\lambda} p_{\rho}}{\sqrt{D}}
$$

Indeed, one can show that these tensors are orthogonal to $p$ and $u$, i.e.,

$$
\begin{aligned}
& e^{\mu \nu} u_{\nu}=e^{\mu \nu} p_{\nu}=0, \\
& \epsilon^{\mu \nu} u_{\nu}=\epsilon^{\mu \nu} p_{\nu}=0 .
\end{aligned}
$$

They also satisfy the relations

$$
\begin{aligned}
& e^{\mu \nu} e_{\nu}{ }^{\beta}=e^{\mu \beta}, \\
& e^{\mu \nu} \epsilon_{\nu}{ }^{\beta}=\epsilon^{\mu \nu} e_{\nu}^{\beta}=\epsilon^{\mu \beta}, \\
& \epsilon^{\mu \nu} \epsilon_{\nu}{ }^{\beta}=-e^{\mu \beta} .
\end{aligned}
$$

Using these properties, one can show that these tensors diagonalize the equation of motion or $\Delta_{\mu \nu}$ in Eq. (3), since

$$
\begin{gathered}
P_{\mu \nu}^{(\lambda)} \eta^{\nu \alpha} P_{\alpha \beta}^{\left(\lambda^{\prime}\right)}=\delta^{\lambda \lambda^{\prime}} P_{\mu \beta}^{(\lambda)}, \\
P_{\mu \nu}^{(\lambda)} \epsilon^{\nu \alpha} P_{\alpha \beta}^{\left(\lambda^{\prime}\right)}=(-i \lambda) \delta^{\lambda \lambda^{\prime}} P_{\mu \beta}^{(\lambda)} .
\end{gathered}
$$

We can define the analogs to the circular polarization vectors

$$
\begin{aligned}
\varepsilon_{\mu}^{(+)} & =\frac{1}{2}\left(e_{\mu}^{(1)}+i e_{\mu}^{(2)}\right), \\
\varepsilon_{\nu}^{(-)} & =\frac{1}{2}\left(e_{\nu}^{(1)}+i e_{\nu}^{(2)}\right),
\end{aligned}
$$

such that

$$
P_{\mu \nu}^{(\lambda)}=-\varepsilon_{\mu}^{(\lambda)}(p) \varepsilon_{\nu}^{*(\lambda)}(p) .
$$


The transverse propagator is

$$
i G_{\mu \nu}^{T}(p)=\sum_{\lambda= \pm}\left(\frac{P_{\mu \nu}^{(\lambda)}}{p^{2}+\lambda a \sqrt{D}}\right)_{p^{2} \rightarrow p^{2}+i \epsilon},
$$

with

$$
a=\frac{4\left(c_{1}(u \cdot p)^{2}-c_{2} u^{2} p^{2}\right)}{M},
$$

where we have included the usual prescription $p^{2} \rightarrow p^{2}+$ $i \epsilon$ to fix the position of the poles in the complex energy plane. This prescription gives the correct position of the poles in the second and fourth quadrants when considering the case $u$ purely spacelike or taking the limit $M \rightarrow \infty$. We use this propagator in the Sec. IV.

\section{PERTURBATIVE GENERATION}

Let us consider the perturbative generation of the MyersPospelov term. One scheme, based on the magnetic coupling, has been developed in Ref. [11], in which it was shown to yield the finite corrections. Here, we deal with another one. Let us consider the fermionic Lagrangian [4], representing itself as an example of a family of Lorentz-breaking higher-derivative fermionic Lagrangians considered in Ref. [14],

$$
\mathcal{L}_{f}=\bar{\psi}\left(i \not D-m+\frac{\eta_{2}}{M} \gamma_{5} \not b(v \cdot D)^{2}\right) \psi,
$$

where $D_{\mu}=\partial_{\mu}-i e A_{\mu}, v_{\mu}$ is a dimensionless vector, $M$ is the Planck mass (as above), and $\eta_{2}$ is some dimensionless number. So, we have the following explicit form of the Lagrangian:

$$
\begin{aligned}
\mathcal{L}_{f}= & \bar{\psi}\left(i \not \partial-m+\frac{\eta_{2}}{M} \gamma_{5} \not(v \cdot \partial)^{2}+e \not A\right. \\
& +\frac{\eta_{2}}{M} \gamma_{5} \not v^{\mu} v^{\nu}\left(-i e\left(A_{\mu} \partial_{\nu}+A_{\nu} \partial_{\mu}\right)\right. \\
- & \left.\left.i e\left(\partial_{\mu} A_{\nu}\right)-e^{2} A_{\mu} A_{\nu}\right)\right) \psi .
\end{aligned}
$$

We note that the similar coupling, but including third derivatives, has been used in Ref. [15].

One can easily observe that the one-loop effective action of second order in $A$, in lower order in $\eta_{2}$, is given by two contributions graphically represented by Fig. 1 . The first of them involves only usual (minimal) vertices proportional to $e$ which do not involve any $v^{\mu}$ vector, and $\eta_{2}$ arises from the expansion of the propagator and is given by graphs $a$ and $b$ of Fig. 1. It looks like

$$
S_{\mathrm{eff}}^{(2)}=\frac{i e^{2}}{2} \int d^{4} x \Pi_{v}^{\mu \nu} A_{\mu} A_{\nu},
$$

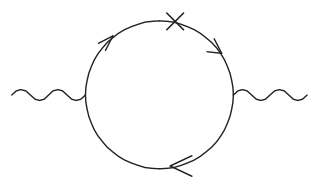

(a)

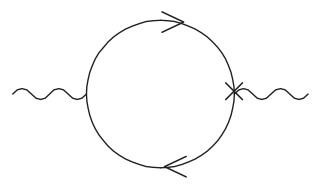

(c)

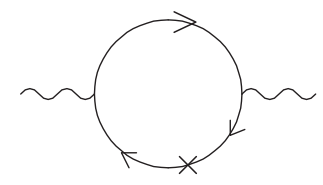

(b)

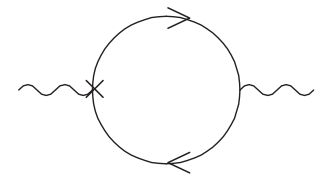

(d)
FIG. 1. The contributions to the two-point function of $A^{\mu}$ with triple vertices.

where

$$
\Pi_{v}^{\mu \nu}=\int \frac{d^{4} p}{(2 \pi)^{4}} \operatorname{tr} G_{v}(p) \gamma^{\mu} G_{v}(p-k) \gamma^{\nu}
$$

with

$$
G_{v}(p)=\frac{1}{\not p-m-\frac{\eta_{2}}{M} \gamma_{5} \not p(v \cdot p)^{2}} .
$$

Now, by applying the expansion

$$
G_{v}(p)=S(p)+S(p) \frac{\eta_{2}}{M} \gamma_{5} \not(v \cdot p)^{2} S(p)+\cdots,
$$

with $S(p)=(\not p-m)^{-1}$, we can easily single out the terms of first order in $\eta_{2}$, by writing

$$
\begin{aligned}
\Pi_{1 M P}^{\mu \nu}(k)= & \frac{\eta_{2}}{M} \mu^{4-D} \int \frac{d^{D} p}{(2 \pi)^{D}} \operatorname{tr}\left[S(p) \gamma_{5} \not(v \cdot p)^{2}\right. \\
& \times S(p) \gamma^{\mu} S(p-k) \gamma^{\nu}+S(p) \gamma^{\mu} S(p-k) \gamma_{5} \\
& \left.\times \not p(v \cdot p-v \cdot k)^{2} S(p-k) \gamma^{\nu}\right],
\end{aligned}
$$

where we have promoted the integral to the $D$-dimensional spacetime, so that $d^{4} p /(2 \pi)^{4}$ is replaced by to $\mu^{4-D} d^{D} p /(2 \pi)^{D}$, with $\mu$ being a renormalization scale.

To calculate the above integrals, we will use the Feynman parametrization, so Eq. (34) is rewritten as

$$
\begin{aligned}
\Pi_{1 M P}^{\mu \nu}(k)= & \frac{\eta_{2}}{M} \mu^{4-D} \int_{0}^{1} d x \int \frac{d^{D} p}{(2 \pi)^{D}} \frac{1}{\left(p^{2}-M_{x}^{2}\right)^{3}} \\
& \times \operatorname{tr}\left[2(1-x)(q+m) \gamma_{5} \not(v \cdot q)^{2}(q+m)\right. \\
& \times \gamma^{\mu}\left(q_{1}+m\right) \gamma^{\nu}+2 x(q+m) \gamma^{\mu}\left(\ell_{1}+m\right) \\
& \left.\times \gamma_{5} \not\left(v \cdot q_{1}\right)^{2}\left(q_{1}+m\right) \gamma^{\nu}\right],
\end{aligned}
$$

where $\quad M_{x}^{2}=m^{2}-(1-x) x k^{2}, \quad q^{\mu}=p^{\mu}+x k^{\mu}, \quad$ and $q_{1}^{\mu}=q^{\mu}-k^{\mu}$. Then, after the calculating the trace, we obtain 


$$
\Pi_{1 M P}^{\mu \nu}(k)=\frac{\eta_{2}}{M} \mu^{4-D} \int_{0}^{1} d x \int \frac{d^{D} p}{(2 \pi)^{D}} \frac{1}{\left(p^{2}-M_{x}^{2}\right)^{3}} \sum_{i=1}^{4} I_{i}^{\mu \nu}
$$

with

$$
\begin{aligned}
I_{1}^{\mu \nu} & =-16 \epsilon^{\mu \alpha \beta \gamma} q_{\alpha} v_{\beta} k_{\gamma}\left[(1-x) q^{\nu}(v \cdot q)^{2}+x q_{1}^{\nu}\left(v \cdot q_{1}\right)^{2}\right], \\
I_{2}^{\mu \nu} & =-16 \epsilon^{\alpha \nu \beta \gamma} q_{\alpha} v_{\beta} k_{\gamma}\left[(1-x) q^{\mu}(v \cdot q)^{2}+x q_{1}^{\mu}\left(v \cdot q_{1}\right)^{2}\right], \\
I_{3}^{\mu \nu} & =8 \epsilon^{\mu \nu \beta \gamma} v_{\beta} k_{\gamma}\left[(1-x)(v \cdot q)^{2}\left(q^{2}-m^{2}\right)+2 x\left(v \cdot q_{1}\right)^{2}\left(q \cdot q_{1}-m^{2}\right)\right], \\
I_{4}^{\mu \nu} & =8 \epsilon^{\mu \nu \alpha \beta} q_{\alpha} v_{\beta}\left[(1-x)(v \cdot q)^{2}\left(q^{2}+m^{2}-2 q \cdot q_{1}\right)-x\left(v \cdot q_{1}\right)^{2}\left(q_{1}^{2}+m^{2}-2 q \cdot q_{1}\right)\right] .
\end{aligned}
$$

In the following, after we integrate over $d^{D} p$ and expand the result around $D=4$, we have

$$
\begin{aligned}
\Pi_{1 M P}^{\mu \nu}= & -\frac{i}{4 \pi} \frac{\eta_{2}}{M} \epsilon^{\mu \nu \beta \gamma} v_{\beta} k_{\gamma} \int_{0}^{1} d x\left(\frac{1}{\epsilon}-\ln \frac{M_{x}}{\mu^{\prime}}\right)\left[\left(10(1-x)^{2} x^{2} k^{2}+(1-6(1-x) x) m^{2}\right) v^{2}\right. \\
& \left.-4(1-x)(2-5(1-x) x) x(v \cdot k)^{2}\right]-\frac{i}{8 \pi} \frac{\eta_{2}}{M} \epsilon^{\mu \nu \beta \gamma} v_{\beta} k_{\gamma} \int_{0}^{1} d x \\
& \times \frac{1}{M_{x}^{2}}\left[(1-6(1-x) x) M_{x}^{4} v^{2}-2(1-x)^{2} x^{2} k^{2}(3-4(1-x) x)(v \cdot k)^{2}\right]
\end{aligned}
$$

where $\epsilon=4-D$ and $\mu^{\prime 2}=4 \pi \mu^{2} e^{-\gamma}$. We note that, as $\int_{0}^{1} d x(1-6(1-x) x)=0$, the divergent contribution does not depend on the mass $m$.

Finally, after we integrate over the parameter $x$, we obtain

$$
\begin{aligned}
\Pi_{1 M P}^{\mu \nu}= & -\frac{1}{12 \pi^{2} \epsilon^{\prime}} \frac{\eta_{2}}{M}\left[k^{2} v^{2}-2(v \cdot k)^{2}\right] \epsilon^{\mu \nu \lambda \rho} n_{\lambda} k_{\rho}+\frac{1}{72 \pi^{2}} \frac{\eta_{2}}{M}\left[5 k^{2}+12 m^{2}+\frac{6\left(k^{4}-2 k^{2} m^{2}-8 m^{4}\right)}{\sqrt{k^{2}\left(4 m^{2}-k^{2}\right)}} \csc ^{-1}\left(\frac{2 m}{\sqrt{k^{2}}}\right)\right] v^{2} \epsilon^{\mu \nu \lambda \rho} v_{\lambda} k_{\rho} \\
& -\frac{1}{18 \pi^{2}} \frac{\eta_{2}}{M}\left[1-\frac{3 m^{2}}{k^{2}}+\frac{3\left(k^{4}-2 k^{2} m^{2}+4 m^{4}\right)}{\sqrt{k^{6}\left(4 m^{2}-k^{2}\right)}} \csc ^{-1}\left(\frac{2 m}{\sqrt{k^{2}}}\right)\right](v \cdot k)^{2} \epsilon^{\mu \nu \lambda \rho} v_{\lambda} k_{\rho},
\end{aligned}
$$

with $\frac{1}{\epsilon^{\prime}}=\frac{1}{\epsilon}-\ln \frac{m}{\mu^{\prime}}$. Here, we can consider the limits $k^{2} \ll m^{2}(m \neq 0)$ and $k^{2} \gg m^{2}(m=0)$ so that we get

$$
\Pi_{1 M P}^{\mu \nu}=-\frac{1}{12 \pi^{2} \epsilon^{\prime}} \frac{\eta_{2}}{M}\left[k^{2} v^{2}-2(v \cdot k)^{2}\right] \epsilon^{\mu \nu \lambda \rho} v_{\lambda} k_{\rho}+\mathcal{O}\left(\frac{k^{2}}{m^{2}}\right)
$$

and

$$
\Pi_{1 M P}^{\mu \nu}=-\frac{1}{12 \pi^{2} \epsilon^{\prime \prime}} \frac{\eta_{2}}{M}\left[k^{2} v^{2}-2(v \cdot k)^{2}\right] \epsilon^{\mu \nu \lambda \rho} v_{\lambda} k_{\rho}+\frac{1}{72 \pi^{2}} \frac{\eta_{2}}{M}\left[5 k^{2} v^{2}-4(v \cdot k)^{2}\right] \epsilon^{\mu \nu \lambda \rho} v_{\lambda} k_{\rho}+\mathcal{O}\left(\frac{m^{2}}{k^{2}}\right),
$$

respectively, where we have also defined $\frac{1}{\epsilon^{\prime \prime}}=\frac{1}{\epsilon}-\ln \frac{k}{\mu^{\prime}}$, with $k=\sqrt{k^{2}}$.

Therefore, for the induced bosonic Myers-Pospelov term from the contribution (40), which corresponds to the nonzero mass, we have

$$
S_{1 M P}=\frac{e^{2}}{12 \pi^{2} \epsilon^{\prime}} \frac{\eta_{2}}{M} \int d^{4} x\left[v^{2} v_{\beta} \epsilon^{\beta \mu \nu \lambda} A_{\mu} \square F_{\nu \lambda}-2 v^{\alpha} F_{\alpha \mu}(v \cdot \partial) v_{\beta} \epsilon^{\beta \mu \nu \lambda} F_{\nu \lambda}\right] .
$$

This enforces the fact that the above higher-derivative terms should be introduced from the very beginning (1) so that we have a consistent subtraction of the divergences.

Then, the quartic vertex (corresponding Feynman diagram is given by Fig. 2) evidently should give a zero contribution. Indeed, this diagram can yield only the Proca-like term $(v \cdot A)^{2}$ with no derivatives, since there are no derivatives of $A_{\mu}$ in the classical action, and the only relevant graph is a tadpole, so the integration over the internal momentum cannot yield a contribution depending on the external momentum, and this term is inconsistent with the gauge symmetry (its vanishing can be shown explicitly, as well). 


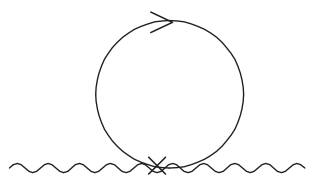

FIG. 2. The contributions to the two-point function of $A^{\mu}$ with the quartic vertex.

To find the remaining first-order contribution in $\eta_{2}$ presented by graphs $c$ and $d$ of Fig. 1, we should consider the contraction of two vertices: the first of them is the usual $e \bar{\psi} A \psi$, and the second one is $-i e \bar{\psi} \frac{\eta_{2}}{M} \gamma_{5} \not v^{\mu} v^{\nu}\left(\left(A_{\mu} \partial_{\nu}+\right.\right.$ $\left.\left.A_{\nu} \partial_{\mu}\right)+\left(\partial_{\mu} A_{\nu}\right)\right) \psi$, where the propagator is the free one [indeed, expanding (32), we will get only the higher-order contributions]. Its explicit form is

$$
\begin{aligned}
S_{2 M P} & =i e^{2} \frac{\eta_{2}}{M} v^{\nu} v^{\mu} A_{\mu}(-k) A_{\lambda}(k) \int \frac{d^{4} p}{(2 \pi)^{4}}\left(2 p_{\nu}+k_{\nu}\right) \\
& \times \operatorname{tr}\left[\gamma_{5} \not \prec \frac{1}{\not p-m} \gamma^{\lambda} \frac{1}{\not p+\not k-m}\right] .
\end{aligned}
$$

Here, the factor $2 p_{\nu}+k_{\nu}$ originates from the nonminimal vertex given by the expression $-i e \bar{\psi} \frac{\eta_{2}}{M} \gamma_{5} \not v^{\mu} v^{\nu}\left(\left(A_{\mu} \partial_{\nu}+\right.\right.$ $\left.\left.A_{\nu} \partial_{\mu}\right)+\left(\partial_{\mu} A_{\nu}\right)\right) \psi$ (the moment $p$ is for the spinor propagator, and the moment $k$ is for external gauge field). It remains to expand this expression up to the third order in external $k$ (actually, the first order in $k$ disappears, so it remains to deal only with the third order). Indeed, the trace in (43) can be calculated before of any expansion of the propagator in series in $k$ :

$$
\begin{aligned}
S_{2 M P}= & -4 e^{2} \frac{\eta_{2}}{M} v^{\nu} v^{\mu} A_{\mu}(-k) A_{\lambda}(k) \epsilon^{\alpha \beta \lambda \rho} \\
& \times \int \frac{d^{4} p}{(2 \pi)^{4}}\left(2 p_{\nu}+k_{\nu}\right) v_{\alpha} p_{\beta} k_{\rho} \\
& \times \frac{1}{\left(p^{2}-m^{2}\right)\left[(p+k)^{2}-m^{2}\right]} .
\end{aligned}
$$

Now, this expression can be expanded into power series in external momenta, where only the third order should be taken into account (the first and second orders evidently vanish; for the first order, one evidently will have the contraction of the Levi-Civita symbol with two Lorentzbreaking vectors, which immediately vanishes, and for the second order, the corresponding scalar simply does not exist).

However, to study it, we can first present it as

$$
S_{2 M P}=A_{\mu}(-k) \Pi_{2 M P}^{\mu \lambda} A_{\lambda}(k),
$$

where

$$
\Pi_{2 M P}^{\mu \lambda}=-4 e^{2} \frac{\eta_{2}}{M} v^{\nu} v^{\mu} \epsilon^{\alpha \beta \lambda \rho} k_{\rho} v_{\alpha} Q_{\nu \beta},
$$

with

$$
Q_{\nu \beta}=\int \frac{d^{4} p}{(2 \pi)^{4}}\left(2 p_{\nu}+k_{\nu}\right) p_{\beta} \frac{1}{\left(p^{2}-m^{2}\right)\left[(p+k)^{2}-m^{2}\right]} .
$$

It is clear that, up to the second order in the external $p$ (the highest relevant order), the tensor $Q_{\nu \beta}$ must look like

$$
Q_{\nu \beta}=Q_{1} \eta_{\nu \beta}+Q_{2} k_{\nu} k_{\beta} .
$$

Indeed, there are no other possible tensor structures. Here, $Q_{1}$ and $Q_{2}$ are two (divergent) constants. Substituting this structure into the contribution (46), we find that it identically vanishes. Hence, this "mixed" contribution is zero, and the only nontrivial result for the Myers-Pospelov term is given by (42). The divergent nature of this result immediately shows that for consistency of the theory one should have the higher-derivative CFJ-like and Myers-Pospelov term presented in the classical action from the very beginning.

Now, we can discuss the renormalization. Actually, our theory is nonrenormalizable (indeed, our coupling is $\frac{\alpha}{M}$, and it has a negative mass dimension; we note that the models considered in Refs. [11,16] allowing for arising the higher-derivative Lorentz-breaking terms are also nonrenormalizable). As is well known, the nonrenormalizable theories are treated as effective ones (see a detailed discussion of the concept of effective field theories in Ref. [17]). They typically arise after integrating over some fields, usually the heavy ones with the characteristic mass $M_{\text {char }}$ of which the role is played in our theory by $M$, in some fundamental, renormalizable theory. As a result, the action of an effective theory represents itself as a power series in $\frac{1}{M_{\text {char }}}$; hence, the coupling constants, being proportional to different positive degrees of $\frac{1}{M_{\text {char }}}$, have negative mass dimension, and the theory turns out to be nonrenormalizable. However, the linearly and quadratically divergent contributions in the effective theories are proportional to positive degrees of the cutoff scale $\mu$, and if $\mu \ll M_{\text {char }}$, these contributions turn out to be strongly suppressed being proportional to $\left(\frac{\mu}{M_{\text {char }}}\right)^{n}$, with $n \geq 1$. Since $\frac{\mu}{M_{\text {char }}} \ll 1$, it is sufficient to restrict the expansion in $\frac{1}{M_{\text {char }}}$ by the first order. This is just the case of our theory, where $M_{\text {char }}=M$ is of the order of the Planck scale, while $\mu$ is naturally estimated to be of the order of $1 \mathrm{TeV}$; see, for example, Ref. [18]. So, we can restrict ourselves by the contributions of the first order in $\frac{1}{M}$.

It is not difficult to show that, in the one-loop approximation, for external $A_{\mu}$, the superficial degree of divergence looks like

$$
\omega=4-V_{1}-2 V_{0 a}-2 V_{0 b}-2 V_{2}-N_{d},
$$

where $V_{1}$ is the number of vertices $\bar{\psi} \gamma_{5} \not v^{\mu} v^{\nu} A_{\mu} \partial_{\nu} \psi, V_{2}$ is the number of vertices $\bar{\psi} \gamma \gamma_{5} \not(v \cdot A)^{2} \psi, V_{0 a}$ is the number of 
standard vertices $\bar{\psi} A \psi, V_{0 b}$ is the number of vertices $\bar{\psi} \gamma_{5} \not v^{\mu} v^{\nu}\left(\partial_{\mu} A_{\nu}\right) \psi$, and $N_{d}$ is the number of derivatives acting to external legs (except those ones in $V_{0 b}$ ). We note that only $V_{0 a}$ vertices are not $\frac{1}{M}$ suppressed. It is clear then that, first, the number of external $A_{\mu}$ legs cannot be less than 2 (hence, $2 V_{2}+V_{0 a}+V_{0 b}+V_{1} \geq 2$ ) and that for gauge symmetry reasons there must be at least one derivative acting to gauge legs (to get the CFJ term) or two derivatives (to get the Maxwell or aether terms). Also, it is evident that the potentially divergent Feynman diagrams with $V_{2}=1,2$ will be not gauge invariant since they will yield the contributions proportional to $(v \cdot A)^{2}$ or $(v \cdot A)^{4}$, and they should vanish in some regularization. Hence, in divergent diagrams one should have $V_{2}=0$. Then, the diagram with $V_{0 a}=2$ has been studied above (42), and the contribution with $V_{0 a}=1$ and $V_{0 b}+V_{1}=1$ is just that one given by (44), and its contribution is zero. In principle, we can also have divergences in contributions to the two-point function formed by only $V_{1}$ and $V_{0 b}$ vertices; however, they are strongly suppressed, being proportional to $\frac{1}{M^{2}}$. We conclude our discussion with the statement that up to the order $M^{-1}$ our results are exact, and the only nontrivial divergent contribution is given by (42).

Therefore, we find that the higher-derivative action given by the sum of (1) and (28) is consistent in the oneloop order. We note that, as usual, if we suggest the gauge field to be a purely external one, the one-loop result is exact.

Now, let us introduce finite temperature. To do it, we apply the Matsubara formalism; i.e., in the integrals over momenta above, Eqs. (34) and (46), we suggest that the zero component of the internal momentum is discrete, $p_{0}=2 \pi T\left(l+\frac{1}{2}\right)$, with $l$ being an integer, and, afterward, we integrate over spatial components of the internal momentum and sum over $l$. As a result, at the finite temperature, our self-energy tensor, given by $\Pi_{M P}^{\mu \nu}=\Pi_{1 M P}^{\mu \nu}+\Pi_{2 M P}^{\mu \nu}$, turns out to look like

$$
\begin{aligned}
\Pi_{M P}^{\mu \nu}= & A(\xi)\left[k^{2} v^{2}-2(v \cdot k)^{2}\right] \epsilon^{\mu \nu \lambda \rho} v_{\lambda} k_{\rho}+B(\xi) k^{2}(v \cdot t)^{2} \epsilon^{\mu \nu \lambda \rho} v_{\lambda} k_{\rho} \\
& -\frac{1}{2} B(\xi) k^{2} v^{2}\left(t^{\mu} t_{\alpha} \epsilon^{\alpha \nu \lambda \rho}+t^{\nu} t_{\alpha} \epsilon^{\mu \alpha \lambda \rho}\right) v_{\lambda} k_{\rho}-2 B(\xi)(k \cdot v)(k \cdot t)(v \cdot t) \epsilon^{\mu \nu \lambda \rho} v_{\lambda} k_{\rho}+B(\xi)(v \cdot k)^{2}\left(t^{\mu} t_{\alpha} \epsilon^{\alpha \nu \lambda \rho}+t^{\nu} t_{\alpha} \epsilon^{\mu \alpha \lambda \rho}\right) v_{\lambda} k_{\rho} \\
& -B(\xi)(k \cdot v)(k \cdot t)\left(v^{\mu} t_{\alpha} \epsilon^{\alpha \nu \lambda \rho}+v^{\nu} t_{\alpha} \epsilon^{\mu \alpha \lambda \rho}\right) v_{\lambda} k_{\rho}+2 B(\xi)(v \cdot k)(v \cdot t)\left(k^{\mu} t_{\alpha} \epsilon^{\alpha \nu \lambda \rho}+k^{\nu} t_{\alpha} \epsilon^{\mu \alpha \lambda \rho}\right) v_{\lambda} k_{\rho} \\
& -\frac{1}{2} B(\xi) k^{2} v^{2}(k \cdot t) \epsilon^{\mu \nu \lambda \rho} v_{\lambda} t_{\rho}+2 B(\xi) k^{2}(k \cdot v)(v \cdot t) \epsilon^{\mu \nu \lambda \rho} v_{\lambda} t_{\rho}+C(\xi) k^{2}(v \cdot t)^{2}\left(t^{\mu} t_{\alpha} \epsilon^{\alpha \nu \lambda \rho}+t^{\nu} t_{\alpha} \epsilon^{\mu \alpha \lambda \rho}\right) v_{\lambda} k_{\rho} \\
& -2 C(\xi)(k \cdot t)^{2} v^{2}\left(t^{\mu} t_{\alpha} \epsilon^{\alpha \nu \lambda \rho}+t^{\nu} t_{\alpha} \epsilon^{\mu \alpha \lambda \rho}\right) v_{\lambda} k_{\rho}-2 C(\xi)(k \cdot t)^{3} v^{2} \epsilon^{\mu \nu \lambda \rho} v_{\lambda} t_{\rho}+C(\xi) k^{2}(k \cdot t)(v \cdot t)^{2} \epsilon^{\mu \nu \lambda \rho} v_{\lambda} t_{\rho} \\
& +D(\xi)(k \cdot t)^{2}(v \cdot t)^{2}\left(t^{\mu} t_{\alpha} \epsilon^{\alpha \nu \lambda \rho}+t^{\nu} t_{\alpha} \epsilon^{\mu \alpha \lambda \rho}\right) v_{\lambda} k_{\rho}+D(\xi)(k \cdot t)^{3}(v \cdot t)^{2} \epsilon^{\mu \nu \lambda \rho} v_{\lambda} t_{\rho}+\mathcal{O}\left(\frac{k^{2}}{m^{2}}\right)
\end{aligned}
$$

where

$$
\begin{gathered}
A(\xi)=-\frac{1}{12 \pi^{2} \epsilon^{\prime}} \frac{\eta_{2}}{M}-\frac{1}{12 \pi^{2}} \frac{\eta_{2}}{M} \int_{|\xi|}^{\infty} d z \frac{(\tanh (\pi z)-1)}{\sqrt{(z-\xi)(\xi+z)}}, \\
B(\xi)=-\frac{1}{6} \frac{\eta_{2}}{M} \int_{|\xi|}^{\infty} d z \sqrt{(z-\xi)(\xi+z)} \tanh (\pi z) \operatorname{sech}^{2}(\pi z), \\
C(\xi)=\frac{1}{12} \frac{\eta_{2}}{M} \int_{|\xi|}^{\infty} d z \frac{\left(\xi^{2}-2 z^{2}\right) \tanh (\pi z) \operatorname{sech}^{2}(\pi z)}{\sqrt{(z-\xi)(\xi+z)}}, \\
D(\xi)=\frac{1}{3} \frac{\eta_{2}}{M} \int_{|\xi|}^{\infty} d z \frac{\tanh (\pi z) \operatorname{sech}^{4}(\pi z)}{\sqrt{(z-\xi)(\xi+z)}}\left(-5 \pi^{2} \xi^{4}+\xi^{2}+\left(5 \pi^{2} \xi^{2}-2\right) z^{2}+\left(\pi^{2} \xi^{4}+\xi^{2}-\left(\pi^{2} \xi^{2}+2\right) z^{2}\right) \cosh (2 \pi z)\right),
\end{gathered}
$$

with $\xi=\frac{m}{2 \pi T}$. Above, we have split the internal momentum as $p^{\mu}=\vec{p}^{\mu}+p_{0} t^{\mu}$, with $\vec{p}^{\mu}=(0, \vec{p})$ and $t^{\mu}=$ $(1,0,0,0)$ being a constant vector along the time axis. We note that these functions of the temperature emerged as well in Ref. [19], in which the finite-temperature extension of results obtained in Ref. [11] for another Lorentz-breaking extension of the QED, involving the magnetic coupling and the coupling of $\psi$ to the constant axial vector $b_{\mu}$, was carried out. It was shown there that in the high-temperature limit all these functions vanish. The result (50) is clearly gauge invariant.

Besides the two-point function of the gauge field, it is important also to consider the two-point function of the spinor field.

Let us calculate this two-point function of the spinor in the first order in $\frac{1}{M}$. We start with the action given by the 


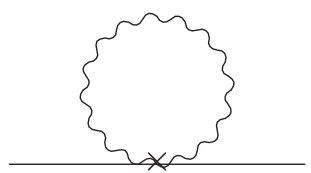

FIG. 3. The contributions to the two-point function of $\psi$ with the quartic vertex.

sum of Lorentz-breaking classical actions (2) and (28), allowing us to obtain the propagators of gauge and spinor fields, respectively. The two-point function of the spinor field is generated by two contributions with external spinor legs: the first one involves two triple vertices, and the second one involves one quartic vertex. To do the calculation, we proceed in the same manner as with the two-point function of the gauge field; that is, we note that the result can be represented in the form of the expansion in $\frac{1}{M}$ and will find the first order in this expansion, just as we have done above. The Lorentz-breaking insertions into the Feynman diagrams below are denoted by the $x$ symbol.
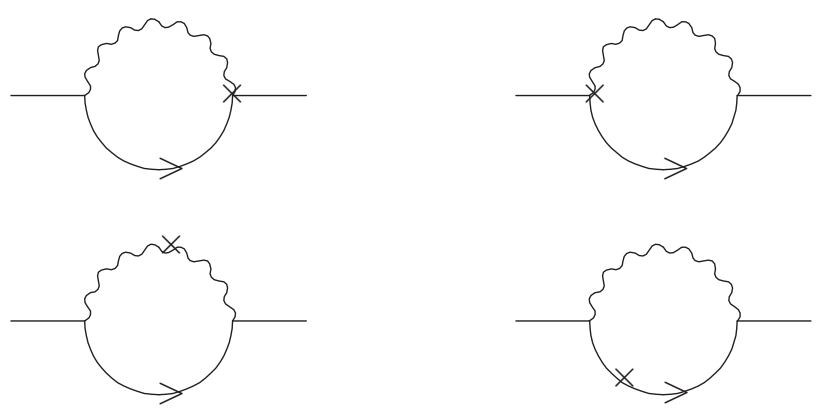

FIG. 4. The contributions to the two-point function of $\psi$ with triple vertices.

We see that, since the quartic vertex is proportional to $\frac{1}{M}$, we can keep in the propagator of the gauge field only the zero-order terms in $\frac{1}{M}$, so we have $\left\langle A^{\mu}(-k) A^{\nu}(k)\right\rangle=\frac{i \eta^{\mu \nu}}{k^{2}}$. Hence, the contribution of the diagram with quartic vertex given by Fig. 3 is proportional to $\int \frac{d^{4} k}{(2 \pi)^{4}} \frac{1}{k^{2}}=0$. As a result, we are left with triple vertices only. They look like

$$
\mathcal{L}_{\text {triple }}=-e \bar{\psi}\left(-\not A+i \frac{\eta_{2}}{M} \gamma_{5} \not v^{\mu} v^{\nu}\left(A_{\mu} \partial_{\nu}+A_{\nu} \partial_{\mu}+\left(\partial_{\mu} A_{\nu}\right)\right)\right) \psi .
$$

The explicit form of the vertices, in the momentum space, is

$$
\begin{aligned}
V_{0}\left(l_{1}, l_{2}, l_{3}\right) & =e \bar{\psi}\left(l_{1}\right) \gamma^{\kappa} \psi\left(l_{2}\right) A_{\kappa}\left(l_{3}\right)(2 \pi)^{4} \delta\left(l_{1}+l_{2}+l_{3}\right), \\
V_{1}\left(k_{1}, k_{2}, k_{3}\right) & =-\frac{e \eta_{2}}{M} v^{\mu} v^{\nu} \bar{\psi}\left(k_{1}\right) \gamma_{5} \not \psi\left(k_{2}\right) A_{\lambda}\left(k_{3}\right)\left[\delta_{\mu}^{\lambda} k_{2 \nu}-\delta_{\nu}^{\lambda} k_{1 \mu}\right](2 \pi)^{4} \delta\left(k_{1}+k_{2}+k_{3}\right) .
\end{aligned}
$$

So, we have the graphs given by Fig. 4. In the two upper graphs of Fig. 4, we consider usual propagators and modified vertices (i.e., one usual vertex $V_{0}$ and one new vertex $V_{1}$ ). The result is, respectively,

$$
\begin{aligned}
& T_{1}(k)=-\frac{e^{2} \eta_{2}}{M} v^{\mu} v^{\nu} \int \frac{d^{4} p}{(2 \pi)^{4}} \bar{\psi}(k) \gamma^{\kappa}(\not p-m) \gamma_{5} \not \phi \psi(k) \eta_{\lambda \kappa}\left[\delta_{\mu}^{\lambda} k_{\nu}-\delta_{\nu}^{\lambda} p_{\mu}\right] \frac{1}{\left(p^{2}+m^{2}\right)(k-p)^{2}}, \\
& T_{2}(k)=-\frac{e^{2} \eta_{2}}{M} v^{\mu} v^{\nu} \int \frac{d^{4} p}{(2 \pi)^{4}} \bar{\psi}(k) \gamma_{5} \not p(\not p-m) \gamma^{\kappa} \psi(k) \eta_{\lambda \kappa}\left[\delta_{\nu}^{\lambda} k_{\mu}-\delta_{\mu}^{\lambda} p_{\nu}\right] \frac{1}{\left(p^{2}+m^{2}\right)(k-p)^{2}} .
\end{aligned}
$$

In the two lower graphs of Fig. 4, we have insertions into the propagators. The graph with an insertion into the gauge propagator, with $\Sigma(k)=-c_{2} u^{2} k^{2}+c_{1}(u \cdot k)^{2}$, is

$$
T_{3}(k)=\frac{4 e^{2} \eta_{2}}{M} \int \frac{d^{4} p}{(2 \pi)^{4}} \bar{\psi}(-k) \gamma^{\mu}(\not p+m) \gamma^{\nu} \psi(k) \frac{\eta_{\nu \alpha} \eta_{\mu \beta}}{(k+p)^{4}} \epsilon^{\alpha \beta \rho \sigma} u_{\rho}\left(p_{\sigma}+k_{\sigma}\right) \frac{\Sigma(k+p)}{\left(p^{2}-m^{2}\right)} \text {. }
$$

And the graph with an insertion into the spinor propagator is

$$
T_{4}(k)=\frac{e^{2} \eta_{2}}{M} \int \frac{d^{4} p}{(2 \pi)^{4}} \bar{\psi}(-k) \gamma^{\mu}(\not p+m) \gamma_{5} \not b(v \cdot p)^{2}(\not p+m) \gamma^{\nu} \psi(k) \frac{\eta_{\mu \nu}}{(k+p)^{2}} \frac{1}{\left(p^{2}-m^{2}\right)^{2}} .
$$

Within the calculation, we expand these contributions up to the second order in the external $k_{\mu}$. It remains to simplify these expressions. Again, we use the dimensional regularization with $\epsilon=4-D$. We take into account only terms up to the second order in external momenta because the higher orders do not contribute to one-loop divergences.

It is instructive here to give some intermediate steps of the calculation. First of all, the structures of $T_{1}$ and $T_{2}$ are rather similar, so that can be summed, and $T_{4}$ can be simplified with the use of the identities $\gamma^{\mu} \gamma^{\nu} \gamma_{\mu}=-2 \gamma^{\nu}$ and $\gamma^{\mu}\left[\gamma^{\alpha}, \gamma^{\beta}\right] \gamma_{\mu}=0$. Then, we have 


$$
\begin{aligned}
T_{1}(k)+T_{2}(k) & =-\frac{2 e^{2} \eta_{2}}{M} \int \frac{d^{4} p}{(2 \pi)^{4}} \bar{\psi}(k) \not p \not \phi \gamma_{5} \psi(k) \frac{v \cdot(p+k)}{\left(p^{2}+m^{2}\right)(k-p)^{2}}, \\
T_{4}(k) & =\frac{2 e^{2} \eta_{2}}{M} \int \frac{d^{4} p}{(2 \pi)^{4}} \bar{\psi}(-k)\left[2(v \cdot p) \not p-\not p\left(p^{2}+m^{2}\right)\right] \gamma_{5} \psi(k) \frac{(v \cdot p)^{2}}{(k+p)^{2}} \frac{1}{\left(p^{2}-m^{2}\right)^{2}} .
\end{aligned}
$$

Integrating over momenta, we arrive at the following pole parts of these contributions up to the second order in the external $k_{\mu}$, either in the massive case, in which one can use expansion in $\frac{k^{2}}{m^{2}}$,

$$
\begin{aligned}
T_{1}(k)+T_{2}(k)= & \frac{i e^{2} \eta_{2}}{48 \pi^{2} \epsilon^{\prime} M} \bar{\psi}(-k)\left[\left(v^{2}\left(k^{2}-3 m^{2}\right)-20(k \cdot v)^{2}\right) \not p+10 v^{2}(k \cdot v) \not h \gamma_{5} \psi(k)\right. \\
& -\frac{i e^{2} \eta_{2}}{576 \pi^{2} M} \bar{\psi}(-k)\left(-5 k^{2} v^{2} \not p-98 v^{2}(k \cdot v) \not k+196(k \cdot v)^{2} \not \partial\right) \gamma_{5} \psi(k)+\frac{3 i e^{2} \eta_{2}}{64 \pi^{2} M} \bar{\psi}(-k) m^{2} v^{2} \not \gamma \gamma_{5} \psi(k) \\
& +\mathcal{O}\left(\frac{k^{2}}{m^{2}}\right)
\end{aligned}
$$

or in the massless limit, in which one uses expansion in $\frac{m^{2}}{k^{2}}$,

$$
\begin{aligned}
T_{1}(k)+T_{2}(k)= & \frac{i e^{2} \eta_{2}}{48 \pi^{2} \epsilon^{\prime \prime} M} \bar{\psi}(-k)\left[\left(v^{2} k^{2}-20(k \cdot v)^{2}\right) \not \gamma^{5}+10 v^{2}(k \cdot v) k \gamma_{5}\right] \psi(k) \\
& +\frac{i e^{2} \eta_{2}}{288 \pi^{2} k^{6} M} \bar{\psi}(-k)\left[62 k^{6} v^{2}(k \cdot v) \not \gamma^{5}+\not \gamma \gamma_{5}\left(8 k^{8} v^{2}-124 k^{6}(k \cdot v)^{2}\right)\right] \psi(k)-\mathcal{O}\left(\frac{m^{2}}{k^{2}}\right) .
\end{aligned}
$$

For $T_{4}$, in the massive and massless cases, respectively, we have

$$
\begin{aligned}
T_{4}(k)= & -\frac{i e^{2} \eta_{2}}{96 \pi^{2} \epsilon^{\prime} M} \bar{\psi}(-k)\left[v^{2}\left(\left(k^{2}-6 m^{2}\right) \not p-6 \not k(k \cdot v)\right)+2(k \cdot v)^{2} \not p\right] \gamma_{5} \psi(k) \\
& \left.-\frac{i e^{2} \eta_{2}}{2304 \pi^{2} M} \bar{\psi}(-k)\left(k^{2} v^{2} \not b-78 v^{2} \not k(k \cdot v)+98(k \cdot v)^{2} \not\right)\right) \gamma_{5} \psi(k)+\frac{7 i e^{2} \eta_{2}}{192 \pi^{2} M} \bar{\psi}(-k) m^{2} v^{2} \not \gamma \gamma_{5} \psi(k)+\mathcal{O}\left(\frac{k^{2}}{m^{2}}\right)
\end{aligned}
$$

or

$$
\begin{aligned}
T_{4}(k)= & -\frac{i e^{2} \eta_{2}}{96 \pi^{2} \epsilon^{\prime \prime} M} \bar{\psi}(-k)\left[v^{2}\left(k^{2} \not p-6 \not k(k \cdot v)\right)+2(k \cdot v)^{2} \not p\right] \gamma_{5} \psi(k) \\
& -\frac{i e^{2} \eta_{2}}{1152 \pi^{2} k^{10} M} \bar{\psi}(-k)\left[k^{2} v^{2}\left(16 k^{10} \not-60 k^{8} \not k(k \cdot v)\right)+2(k \cdot v)^{2}\left(22 k^{10} \not--36 k^{8} \not k(k \cdot v)\right)\right] \gamma_{5} \psi(k)+\mathcal{O}\left(\frac{m^{2}}{k^{2}}\right) .
\end{aligned}
$$

The $T_{3}$ has a structure different from $T_{1}, T_{2}, T_{4}$, being proportional to the Levi-Civita symbol. After the integrations over momenta, we find for massive and massless cases, respectively,

$$
\begin{aligned}
T_{3}(k)= & -\frac{i e^{2} \eta_{2}}{96 \pi^{2} \epsilon^{\prime} M} u^{\kappa} \epsilon_{\kappa \lambda \mu \nu} \bar{\psi}(-k)\left[-\gamma^{\mu} \gamma^{\nu} \gamma^{\lambda}\left(u^{2}\left(c_{1}\left(2 m^{2}-k^{2}\right)+4 c_{2}\left(k^{2}-3 m^{2}\right)\right)+2 c_{1}(k \cdot u)^{2}\right)\right. \\
& \left.+\left(2 u^{2}\left(\left(c_{1}-4 c_{2}\right) \not k+2\left(c_{1}-6 c_{2}\right) m\right)+4 c_{1}(k \cdot u) \not l\right) k^{\lambda} \gamma^{\mu} \gamma^{\nu}\right] \psi(k) \\
& -\frac{i e^{2} \eta_{2}}{2304 \pi^{2} M} \bar{\psi}(-k) \gamma^{\mu} \gamma^{\nu} \gamma^{\lambda} \psi(k)\left(-40 c_{2} k^{2} u^{2}+13 c_{1} k^{2} u^{2}-14 c_{1}(k \cdot u)^{2}\right) u^{\kappa} \epsilon_{\kappa \lambda \mu \nu} \\
& -\frac{i e^{2} \eta_{2}}{1152 \pi^{2} M}\left(13 c_{1}-40 c_{2}\right) u^{2} u^{\kappa} k^{\lambda} \epsilon_{\kappa \lambda \mu \nu} \bar{\psi}(-k) \not k \gamma^{\mu} \gamma^{\nu} \psi(k)+\frac{7 i c_{1} e^{2} \eta_{2}}{576 \pi^{2} M}(k \cdot u) u^{\kappa} k^{\lambda} \epsilon_{\kappa \lambda \mu \nu} \bar{\psi}(-k) \not k \gamma^{\mu} \gamma^{\nu} \psi(k) \\
& -\frac{i e^{2} \eta_{2}}{288 \pi^{2} M}\left(5 c_{1}-18 c_{2}\right) m u^{2} \bar{\psi}(-k) \gamma^{\mu} \gamma^{\nu} \psi(k) u^{\kappa} k^{\lambda} \epsilon_{\kappa \lambda \mu \nu} \\
& +\frac{i e^{2} \eta_{2}}{576 \pi^{2} M}\left(11 c_{1}-54 c_{2}\right) m^{2} u^{2} \bar{\psi}(-k) \gamma^{\mu} \gamma^{\nu} \gamma^{\lambda} \psi(k) u^{\kappa} \epsilon_{\kappa \lambda \mu \nu}+\mathcal{O}\left(\frac{k^{2}}{m^{2}}\right)
\end{aligned}
$$

or 


$$
\begin{aligned}
T_{3}(k)= & -\frac{i e^{2} \eta_{2}}{96 \pi^{2} \epsilon^{\prime} M} u^{\kappa} \epsilon_{\kappa \lambda \mu \nu} \bar{\psi}(-k)\left[-\gamma^{\mu} \gamma^{\nu} \gamma^{\lambda}\left(u^{2}\left(-c_{1}+4 c_{2}\right) k^{2}+2 c_{1}(k \cdot u)^{2}\right)\right. \\
& \left.+k^{\lambda}\left(2 u^{2}\left(c_{1}-4 c_{2}\right) \not k+4 c_{1}(k \cdot u) \not l\right) \gamma^{\mu} \gamma^{\nu}\right] \psi(k) \\
& -\frac{i e^{2} \eta_{2}}{576 \pi^{2} k^{10} M} u^{\kappa} k^{\lambda} \epsilon_{\kappa \lambda \mu \nu}\left(k^{2} u^{2}\left(10 c_{1} k^{8}-40 c_{2} k^{8}\right)+12 c_{1} k^{8}(k \cdot u)^{2}\right) \bar{\psi}(-k) \not k \gamma^{\mu} \gamma^{\nu} \psi(k) \\
& +\frac{i e^{2} \eta_{2}}{1152 \pi^{2} k^{8} M} \bar{\psi}(-k) \gamma^{\mu} \gamma^{\nu} \gamma^{\lambda} \psi(k) u^{\kappa} \epsilon_{\kappa \lambda \mu \nu}\left(k^{2} u^{2}\left(-16 c_{1} k^{8}+64 c_{2} k^{8}\right)+20 c_{1} k^{8}(k \cdot u)^{2}\right) \\
& +\frac{5 i c_{1} e^{2} \eta_{2}}{144 \pi^{2} M}(k \cdot u) u^{\kappa} k^{\lambda} \epsilon_{\kappa \lambda \mu \nu} \bar{\psi}(-k) \not l \gamma^{\mu} \gamma^{\nu} \psi(k)+\mathcal{O}\left(\frac{m^{2}}{k^{2}}\right) .
\end{aligned}
$$

However, the form of $T_{3}$ can be presented in the manner similar to $T_{1}, T_{2}, T_{4}$, with the use of the identities,

$$
\sigma^{\mu \nu} \gamma_{5}=\frac{i}{2} \epsilon^{\mu \nu \alpha \beta} \sigma_{\alpha \beta}, \quad \epsilon_{\kappa \lambda \mu \nu} \gamma^{\mu} \gamma^{\nu}=-2 \sigma_{\kappa \lambda} \gamma_{5}, \quad \epsilon_{\kappa \lambda \mu \nu} \gamma^{\lambda} \gamma^{\mu} \gamma^{\nu}=-6 i \gamma_{5} \gamma_{\kappa}
$$

which implies, at $m \neq 0$,

$$
\begin{aligned}
T_{3}(k)= & -\frac{e^{2} \eta_{2}}{96 \pi^{2} \epsilon^{\prime} M} \bar{\psi}(-k)\left[6 \gamma_{5} \not h\left(u^{2}\left(c_{1}\left(2 m^{2}-k^{2}\right)+4 c_{2}\left(k^{2}-3 m^{2}\right)\right)+2 c_{1}(k \cdot u)^{2}\right)\right. \\
& \left.-2 i\left[2 u^{2}\left(\left(c_{1}-4 c_{2}\right) \not k+2\left(c_{1}-6 c_{2}\right) m\right)+4 c_{1}(k \cdot u) \not \iota\right] u^{\kappa} k^{\lambda} \sigma_{\kappa \lambda} \gamma_{5}\right] \psi(k) \\
& -\frac{e^{2} \eta_{2}}{384 \pi^{2} M} \bar{\psi}(-k) \gamma_{5} \not h \psi(k)\left(\left(13 c_{1}-40 c_{2}\right) k^{2} u^{2}-14 c_{1}(k \cdot u)^{2}\right) \\
& +i \frac{e^{2} \eta_{2}}{576 \pi^{2} M}\left(13 c_{1}-40 c_{2}\right) u^{2} u^{\kappa} k^{\lambda} \bar{\psi}(-k) \not k \sigma_{\kappa \lambda} \gamma_{5} \psi(k)-\frac{7 i c_{1} e^{2} \eta_{2}}{288 \pi^{2} M}(k \cdot u) u^{\kappa} k^{\lambda} \bar{\psi}(-k) \not l \sigma_{\kappa \lambda} \gamma_{5} \psi(k) \\
& +\frac{i e^{2} \eta_{2}}{144 \pi^{2} M}\left(5 c_{1}-18 c_{2}\right) m u^{2} \bar{\psi}(-k) \sigma_{\kappa \lambda} \gamma_{5} \psi(k) u^{\kappa} k^{\lambda}+\frac{e^{2} \eta_{2}}{96 \pi^{2} M}\left(11 c_{1}-54 c_{2}\right) m^{2} u^{2} \bar{\psi}(-k) \gamma_{5} \not h \psi(k)+\mathcal{O}\left(\frac{k^{2}}{m^{2}}\right)
\end{aligned}
$$

and, at $m \rightarrow 0$

$$
\begin{aligned}
T_{3}(k)= & -\frac{e^{2} \eta_{2}}{96 \pi^{2} \epsilon^{\prime} M} \bar{\psi}(-k)\left[6 \gamma_{5} \not h\left(u^{2}\left(-c_{1}+4 c_{2}\right) k^{2}+2 c_{1}(k \cdot u)^{2}\right)\right. \\
& \left.-4 i u^{\kappa} k^{\lambda}\left(u^{2}\left(\left(c_{1}-4 c_{2}\right) \not k\right)+4 c_{1}(k \cdot u) \not l\right) \sigma_{\kappa \lambda} \gamma_{5}\right] \psi(k) \\
& +\frac{i e^{2} \eta_{2}}{288 \pi^{2} k^{10} M} u^{\kappa} k^{\lambda}\left(k^{2} u^{2}\left(10 c_{1} k^{8}-40 c_{2} k^{8}\right)+12 c_{1} k^{8}(k \cdot u)^{2}\right) \bar{\psi}(-k) \not k \sigma_{\kappa \lambda} \gamma_{5} \psi(k) \\
& \left.-\frac{e^{2} \eta_{2}}{192 \pi^{2} k^{8} M} \bar{\psi}(-k)\right) \not h \gamma_{5} \psi(k)\left(k^{2} u^{2}\left(-16 c_{1} k^{8}+64 c_{2} k^{8}\right)+20 c_{1} k^{8}(k \cdot u)^{2}\right) \\
& -\frac{5 i c_{1} e^{2} \eta_{2}}{72 \pi^{2} M}(k \cdot u) u^{\kappa} k^{\lambda} \bar{\psi}(-k) \not h \sigma_{\kappa \lambda} \gamma_{5} \psi(k)+\mathcal{O}\left(\frac{m^{2}}{k^{2}}\right)
\end{aligned}
$$

More simplifications are possible in terms involving $\sigma_{\nu \lambda}$ matrices, due to symmetrization by the rules like $\not k \not k=k^{2}, \not \not \not b=v^{2}$, and then, $u^{\kappa} k^{\lambda} \not k \sigma_{\kappa \lambda}=i\left[(u \cdot k) \not k-\not h k^{2}\right]$, and $u^{\kappa} k^{\lambda} \not \iota \sigma_{\kappa \lambda}=i\left[u^{2} \not k-\not h(u \cdot k)\right]$. Thus, we find 


$$
\begin{aligned}
T_{3}(k)= & -\frac{e^{2} \eta_{2}}{96 \pi^{2} \epsilon^{\prime} M} \bar{\psi}(-k)\left[6 \not h\left(u^{2}\left(c_{1}\left(2 m^{2}-k^{2}\right)+4 c_{2}\left(k^{2}-3 m^{2}\right)\right)+2 c_{1}(k \cdot u)^{2}\right)\right. \\
& \left.+4 u^{2}\left(\left(c_{1}-4 c_{2}\right)\left[(u \cdot k) \not k-\not h k^{2}\right]+2 i\left(c_{1}-6 c_{2}\right) m u^{\kappa} k^{\lambda} \sigma_{\kappa \lambda}\right)+4 c_{1}(k \cdot u)\left[u^{2} \not k-\not h(u \cdot k)\right]\right] \gamma_{5} \psi(k) \\
& -\frac{e^{2} \eta_{2}}{384 \pi^{2} M} \bar{\psi}(-k) \gamma_{5} \not h \psi(k)\left(\left(13 c_{1}-40 c_{2}\right) k^{2} u^{2}-14 c_{1}(k \cdot u)^{2}\right)-\frac{e^{2} \eta_{2}}{576 \pi^{2} M}\left(13 c_{1}-40 c_{2}\right) u^{2} \bar{\psi}(-k)\left[(u \cdot k) \not k-\not h k^{2}\right] \gamma_{5} \psi(k) \\
& +\frac{7 c_{1} e^{2} \eta_{2}}{288 \pi^{2} M}(k \cdot u) \bar{\psi}(-k)\left[u^{2} \not k-\not h(u \cdot k)\right] \gamma_{5} \psi(k)+\frac{i e^{2} \eta_{2}}{144 \pi^{2} M}\left(5 c_{1}-18 c_{2}\right) m u^{2} \bar{\psi}(-k) \sigma_{\kappa \lambda} \gamma_{5} \psi(k) u^{\kappa} k^{\lambda} \\
& +\frac{e^{2} \eta_{2}}{96 \pi^{2} M}\left(11 c_{1}-54 c_{2}\right) m^{2} u^{2} \bar{\psi}(-k) \gamma_{5} \not h \psi(k)+\mathcal{O}\left(\frac{k^{2}}{m^{2}}\right)
\end{aligned}
$$

and, at $m \rightarrow 0$,

$$
\begin{aligned}
T_{3}(k)= & -\frac{e^{2} \eta_{2}}{96 \pi^{2} \epsilon^{\prime} M} \bar{\psi}(-k)\left[6 \not h\left(u^{2}\left(-c_{1}+4 c_{2}\right) k^{2}+2 c_{1}(k \cdot u)^{2}\right)+4 u^{2}\left(c_{1}-4 c_{2}\right)\left[(u \cdot k) \not k-\not h k^{2}\right]\right. \\
& \left.+4 c_{1}(k \cdot u)\left[u^{2} \not k-\not h(u \cdot k)\right]\right] \gamma_{5} \psi(k)-\frac{e^{2} \eta_{2}}{288 \pi^{2} k^{2} M}\left(k^{2} u^{2}\left(10 c_{1}-40 c_{2}\right)+12 c_{1}(k \cdot u)^{2}\right) \bar{\psi}(-k)\left[(u \cdot k) \not k-\not k k^{2}\right] \gamma_{5} \psi(k) \\
& \left.-\frac{e^{2} \eta_{2}}{192 \pi^{2} M} \bar{\psi}(-k)\right) \not h \gamma_{5} \psi(k)\left(k^{2} u^{2}\left(-16 c_{1}+64 c_{2}\right)+20 c_{1}(k \cdot u)^{2}\right) \\
& +\frac{5 c_{1} e^{2} \eta_{2}}{72 \pi^{2} M}(k \cdot u) \bar{\psi}(-k)\left[u^{2} \not k-\not h(u \cdot k)\right] \gamma_{5} \psi(k)+\mathcal{O}\left(\frac{m^{2}}{k^{2}}\right)
\end{aligned}
$$

Taking all together, we find that, to achieve multiplicative renormalizability, in the $m \neq 0$ case, the total free Lorentzbreaking Lagrangian of the spinor, corresponding to pole parts of $T_{1}, T_{2}, T_{3}, T_{4}$ together plus the classical action, must be

$$
\begin{aligned}
\mathcal{L}_{\text {total }}= & \bar{\psi}(i \not \partial-m) \psi+\frac{1}{M} \bar{\psi}\left(C_{1} v^{2} \not \square+C_{2}(v \cdot \partial)^{2} \not \gamma+C_{3} v^{2}(v \cdot \partial) \not \partial+C_{4} m^{2} v^{2} \not \gamma+C_{5} m v^{2} \sigma_{\lambda \rho} v^{\lambda} \partial^{\rho}\right) \gamma_{5} \psi \\
& +\left(C_{i} \rightarrow C_{i}^{\prime}, v^{\mu} \rightarrow u^{\mu}\right),
\end{aligned}
$$

where $C_{1} \ldots C_{5}, C_{1}^{\prime} \ldots C_{5}^{\prime}$ are dimensionless constants, and each term of the given dependence in $v^{\mu}$ has its analog where $v^{\mu}$ is replaced by the $u^{\mu}$. In our case, the last term proportional to $C_{5}^{\prime}$ emerges only with the $u^{\mu}$ vector, arising from $T_{3}$, and there is no term proportional to $C_{5}$. We note that, as frequently occurs, the Lorentz-breaking vectors are lightlike, and some terms in quantum corrections simply vanish, so the structure of quantum corrections simplifies drastically (for example, a similar situation takes place in Ref. [11]). Namely, if both $v^{2}=0$ and $u^{2}=0$, this Lagrangian exactly matches the kinetic part of the Lagrangian (28), which we used as a starting point. We note that, for dimensional and symmetry reasons, it is easy to conclude that the same quantum corrections (72) will emerge if, instead of (28), we used the gauge extension of (72). Also, the new terms proportional to $\frac{m}{M} u^{2} \bar{\psi} \gamma \gamma_{5}(u \cdot \partial) \psi$ or its analog in which $u^{\mu}$ is replaced by $v^{\mu}$, can arise in these cases.

We note that, in principle, the explicit results of integration over momenta can be obtained as well in general case, without imposing any of these limits; however, they are extremely cumbersome. It is interesting to observe that if the Lorentz-breaking vectors $u^{\mu}$ and $v^{\mu}$ are lightlike the zero and first orders in external momenta in these contributions vanish.

The whole contribution to the two-point function of the spinor is given by the sum of $T_{1}, T_{2}, T_{3}$, and $T_{4}$ : in the massive case, one finds a sum of (61), (70), (63), and in the massless case, one finds the sum of (62), (71), (64). We close the section with the conclusion that we found the twopoint functions in both the gauge and matter sectors of our extension of the QED.

\section{UNITARITY ASPECTS IN THE EXTENDED QED}

It is well known that the presence of higher time derivatives in quantum field theory can lead to an indefinite metric in Hilbert space. The sector with a negative metric of the theory produces negative norm states or ghosts, which introduce several conceptual issues in connection with the conservation of probability or unitarity. However, in the subclass of higher time derivative theories called Lee-Wick theories, in which the additional d.o.f. arise in complex conjugate poles, perturbative unitarity has been well 
established [20]. The idea is that, since the structure of poles determines the discontinuities in phase space, under some assumptions, both contributions of complex conjugate modes cancel each other order by order in the perturbative series [21]. The issue of analyticity in the complex energy plane and the resulting cutting equations have been intensively studied over the past years (for the general discussion of ghost states, see, for example, Ref. [22]). The Lee-Wick prescription of removing the negative metric particles from the asymptotic space has been shown to be an efficient tool in providing a unitary theory together with the expected convergence property.

In general, to study unitarity in higher time derivatives theories, one is confronted with the problem of analyticity of amplitude diagrams. The direct application of the $i \epsilon$ prescription in the propagators seems to fail to preserve unitarity in many cases; therefore, it is necessary to analyze the configuration of poles case by case. Moreover, the presence of Lorentz symmetry breaking makes the study of analyticity of integrals be more involved. In many cases, there can be an arbitrary number of extra poles associated to negative-metric states, which marks a departure with respect to the pole structure of a Lee-Wick theory that one uses to prove unitarity. It is also difficult to consider the perturbative solutions which can become complex under certain conditions, and the corresponding dispersion relation can be extremely difficult to solve [23]. An early approach to deal with analytic properties of phase space integrals in the presence of Lorentz violation, based on the Euclidean space or Wick rotation, is presented in Ref. [24]. Recently, a new formulation for Lee-Wick theories as nonanalytical Euclidean theories was proposed in Refs. $[25,26]$. We follow similar lines to deal with unitarity in our higher time derivative Lorentz-violating model. The strategy we pursue to compute the relevant contributions of discontinuities is to consider the Euclidean theory from the beginning and perform the Wick rotation together with rotation of the preferred 4-vector and to apply the Lee-Wick prescription in cut integrals [20]. In this way, we arrive at the simplified integral with simplified poles.

The processes we study are the Bhabha scattering at tree level (we note that some earlier studies of Bhabha scattering in a Lorentz-breaking extension of QED were carried out in Ref. [27], in which, however, no higher-derivative terms were studied) and Compton scattering at the one-loop level. In the first case, we let the preferred 4-vector to be the most general one, allowing additional d.o.f. and the negative metric to arise, and in the second one, we choose a purely timelike preferred 4-vector without ghosts in the theory. For both cases, we consider the forward scattering of two particles with incoming momenta $p=k$ and $p^{\prime}$ related as

$$
p+p^{\prime} \rightarrow p+p^{\prime}
$$

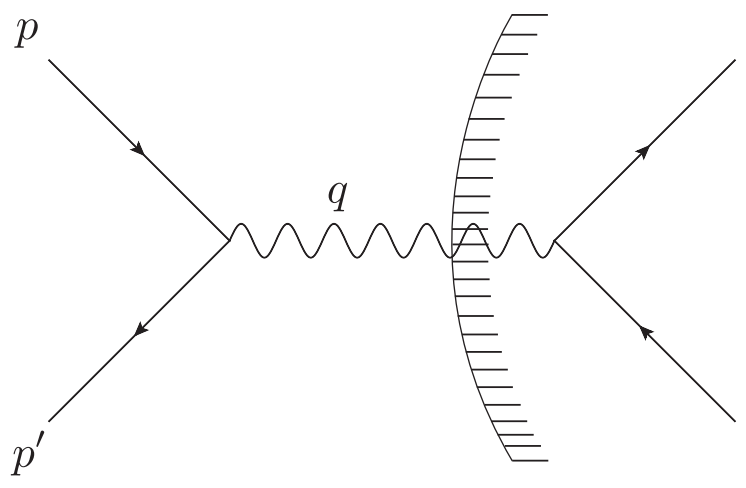

FIG. 5. The Bhabha scattering diagram at tree level.

\section{A. Bhabha scattering at tree level in the ghost sector}

We consider a generic preferred 4-vector $u_{\mu}$ so that, in general, ghosts can arise. We also consider the Bhabha scattering process at tree level given by Fig. 5 .

The amplitude in the transverse gauge is given by

$$
\begin{aligned}
i \mathcal{M}= & (-i e) V^{\mu}\left(p, p^{\prime}\right) \times\left.\sum_{\lambda} \frac{-i P_{\mu \nu}^{(\lambda)}(q)}{\Lambda^{(\lambda)}(q)}\right|_{q=p-p^{\prime}} \\
& \times(-i e) V^{* \nu}\left(p, p^{\prime}\right),
\end{aligned}
$$

where $\Lambda^{(\lambda)}(q)=q^{2}+\lambda a \sqrt{D(q)}$, with $D(q)$ and $a$ given by (10) and (27), respectively, and

$$
\begin{aligned}
V^{\mu}\left(p, p^{\prime}\right) & =\bar{u}(p) \gamma^{\mu} v\left(p^{\prime}\right), \\
V^{* \nu}\left(p, p^{\prime}\right) & =\bar{v}\left(p^{\prime}\right) \gamma^{\nu} u(p) .
\end{aligned}
$$

The standard way to compute the imaginary part of the amplitude in Eq. (74) is to fix the 4-vector $u^{\mu}$, solve the dispersion relation, and, afterward, analyze discontinuities of $\mathcal{M}(s)$, which is an analytic function of the complex variable $s$. However, in our model with modified photons, the dispersion relation is a very complicated expression, and the solutions can be difficult to find. So, we introduce a novel method to deal with unitarity.

The strategy is to start with a theory in Minkowski space, which is defined as the one obtained from the Wick rotation in the Euclidean theory, perhaps nonanalytically, as in Refs. [25,26]. This starting point ensures a well-defined Wick rotation to the Euclidean space warranted by the position of positive and negative poles in the fourth and second quadrants of the energy plane, respectively. Hence, we perform the Wick rotation, changing external momenta $s_{4}=i s_{0}$ so that the dispersion relation decouples into usual and ghost solutions. This last step simplifies the calculation considerably. The rotated energy integral will still depend on the ie prescription, which allows us to compute the discontinuity. Finally, we perform the polarization sum and conveniently evaluate with the delta function in some parts of the integral. Only at the final step, we remove the $i \epsilon$ prescription performing the limit $\epsilon \rightarrow 0$. 
From Eq. (74), we can write

$$
\mathcal{M}(s)=e^{2} V^{\mu} V^{* \nu} \int \frac{d^{4} q}{(2 \pi)^{4}}\left[\sum_{\lambda} \frac{e_{\mu \nu}+i \lambda \epsilon_{\mu \nu}}{2 \Lambda^{(\lambda)}(q)}\right]_{q^{2} \rightarrow q^{2}+i \varepsilon} \delta^{(4)}(q-s),
$$

where we have defined $s=p-p^{\prime}$, included the $i \epsilon$ prescription, and used $P_{\mu \nu}^{(\lambda)}=\frac{1}{2}\left(e_{\mu \nu}+i \lambda \epsilon_{\mu \nu}\right)$. Using the expressions

$$
\frac{1}{\Lambda^{(+)}}+\frac{1}{\Lambda^{(-)}}=\frac{2 q^{2}}{\left(q^{2}\right)^{2}-a^{2} D}, \quad \frac{1}{\Lambda^{(+)}}-\frac{1}{\Lambda^{(-)}}=-\frac{2 a \sqrt{D}}{\left(q^{2}\right)^{2}-a^{2} D}
$$

and (18) and (19), we can write

$$
\mathcal{M}(s)=e^{2} V^{\mu} V^{* \nu} \times \int \frac{d^{4} q}{(2 \pi)^{4}}\left[\frac{1}{\left(q^{2}\right)^{2}-a^{2} D}\left(-q^{2} \eta_{\mu \nu}-\frac{\left(q^{2}\right)^{2}}{D} u_{\mu} u_{\nu}+a i \epsilon_{\mu \alpha \beta \nu} u^{\alpha} q^{\beta}\right)\right]_{q^{2} \rightarrow q^{2}+i \epsilon} \delta^{(4)}(q-s),
$$

where the terms in (75) proportional to $\not{q}=\not p-\not p^{\prime}$ vanish due to the external on-shell spinors.

The Wick rotation has to be done carefully, since the direct analytical extension of the momentum variable in the delta can lead to inconsistencies. The best way to proceed for our integral is to perform the analytic extension in the original expression (74) and then go back with the integral in Eq. (75). However, for an intermediate step, we will extend the delta to complex variables [26]. Before doing this, however, we should mention that solutions in Euclidean space may differ from those in Lorentzian space, so the equivalence of both methods holds with respect to the type of solutions, which eventually propagate through the cuts. Along these steps, by performing the analytic extension with the rule $s_{0}=-i s_{4}$ and momenta $s_{E}=\left(\vec{s}, s_{4}\right)$, we arrive at

$$
\mathcal{M}\left(s_{E}\right)=e^{2} V^{\mu} V^{* \nu} \times \int \frac{d^{4} q_{E}}{(2 \pi)^{4}}\left[\frac{1}{q_{E}^{2}\left(1+\beta^{2} \gamma q_{E}^{2}\right)}\left(\eta_{\mu \nu}+\frac{1}{\gamma} u_{\mu}^{E} u_{\nu}^{E}+\beta i \epsilon_{\mu \alpha \beta \nu} u_{E}^{\alpha} q_{E}^{\beta}\right)\right]_{q_{E}^{2} \rightarrow q_{E}^{2}-i \epsilon} \delta^{(3)}\left(\vec{q}-\vec{p}-\vec{p}^{\prime}\right) \delta\left(q_{4}-s_{4}\right),
$$

with

$$
D_{E}=-\gamma q_{E}^{2}, \quad a_{E}=\beta q_{E}^{2}, \quad \gamma=u_{E}^{2} \sin ^{2} \theta, \quad \beta=\frac{4 u_{E}^{2}\left(c_{1} \cos ^{2} \theta-c_{2}\right)}{M},
$$

where $\theta$ is the angle between the two Euclidean 4-vectors $u_{E}$ and $q_{E}$. Now, in terms of $\varepsilon_{E \nu}^{(\lambda)}$, which is a function of $q_{4}$, we can write Eq. (78) as

$$
\mathcal{M}\left(s_{E}\right)=e^{2} V^{\mu} V^{* \nu} \times \int \frac{d^{4} q_{E}}{(2 \pi)^{4}}\left(\sum_{\lambda} \frac{\left(\varepsilon_{E \mu}^{(\lambda)} \varepsilon_{E \nu}^{*(\lambda)}\left(-1+i \lambda \beta \sqrt{\gamma q_{E}^{2}}\right)\right)_{q_{4}=s_{4}} \delta\left(q_{4}-s_{4}\right)}{\left(q_{E}^{2}-i \epsilon\right)\left(1+\beta^{2} \gamma q_{E}^{2}-i \epsilon\right)}\right) \delta^{(3)}\left(\vec{q}-\vec{p}-\vec{p}^{\prime}\right) .
$$

Also, let us write the denominator in (80) as

$$
\frac{m_{\Lambda}^{2}}{\left(q_{E}^{2}-i \epsilon\right)\left(m_{\Lambda}^{2}+q_{E}^{2}-i \epsilon\right)}=\frac{1}{\left(q_{E}^{2}-i \epsilon\right)}-\frac{1}{\left(q_{E}^{2}+m_{\Lambda}^{2}-i \epsilon\right)},
$$

where $\left(\beta^{2} \gamma\right)^{-1}=m_{\Lambda}^{2}$. We identify two solutions

$$
\omega=|\vec{q}|, \quad W=\sqrt{|\vec{q}|^{2}+m_{\Lambda}^{2}},
$$

where the first solution is for the standard photon, and the second one, which arising at a higher scale $m_{\Lambda} \sim M$, is for a massive ghost. 
To compute the discontinuities in terms of $s_{4}$, we focus on the element

$$
F\left(s_{4}\right)=\frac{1}{\left(q_{E}^{2}-i \epsilon\right)}-\frac{1}{\left(q_{E}^{2}+m_{\Lambda}^{2}-i \epsilon\right)}
$$

and rewrite it as

$$
F\left(s_{4}\right)=\frac{1}{\left(s_{4}^{2}+\omega^{2}-i \epsilon\right)}-\frac{1}{\left(s_{4}^{2}+W^{2}-i \epsilon\right)}
$$

Now, we decompose each term as

$$
\begin{aligned}
\frac{1}{\left(s_{4}^{2}+x^{2}-i \epsilon\right)} & =\frac{1}{2 i x}\left[\frac{1}{\left(s_{4}-i x-\epsilon\right)}-\frac{1}{\left(s_{4}+i x+\epsilon\right)}\right] \\
& =\frac{1}{2 x}\left[\frac{1}{\left(i s_{4}+x-i \epsilon\right)}-\frac{1}{\left(i s_{4}-x+i \epsilon\right)}\right],
\end{aligned}
$$

where some $\epsilon$ terms have been neglected in the numerator. Next, we introduce the extended delta to complex variables

$$
\lim _{\epsilon \rightarrow 0}\left[\frac{1}{z-i \epsilon}-\frac{1}{z+i \epsilon}\right]=2 i \pi \tilde{\delta}(z)
$$

meaning that it vanishes everywhere except at some value at the real axis, where it reduces to the standard delta function [26]. Applied to our case, we arrive at

$$
\begin{aligned}
\operatorname{Disc}\left[F\left(s_{4}\right)\right]= & \frac{2 i \pi\left(\tilde{\delta}\left(i s_{4}+\omega\right)+\tilde{\delta}\left(i s_{4}-\omega\right)\right)}{2 \omega} \\
& -\frac{2 i \pi\left(\tilde{\delta}\left(i s_{4}+W\right)+\tilde{\delta}\left(i s_{4}-W\right)\right)}{2 W} .
\end{aligned}
$$

Since at effective energies the external momenta are always much less than the high-energy scale defined by $M$, we set the two last delta functions to zero. From the expression (87), one has

$$
\operatorname{Disc}\left[F\left(s_{4}\right)\right]=\frac{2 i \pi\left(\tilde{\delta}\left(i s_{4}+\omega\right)+\tilde{\delta}\left(i s_{4}-\omega\right)\right)}{2 \omega} .
$$

Substituting this expression into Eq. (80), we find

$$
\begin{aligned}
\operatorname{Disc}\left[\mathcal{M}\left(s_{E}\right)\right]= & e^{2} V^{\mu} V^{* \nu} \times \int \frac{d^{4} q_{E}}{(2 \pi)^{4}}\left(\sum_{\lambda} \frac{\left(\varepsilon_{E \mu}^{(\lambda)} \varepsilon_{E \nu}^{*(\lambda)}\left(-1+i \lambda \beta \sqrt{\gamma q_{E}^{2}}\right)\right)_{q_{4}=s_{4}}}{\beta^{2} \gamma} \times \frac{(-2 i \pi)\left(\tilde{\delta}\left(i s_{4}+\omega\right)+\tilde{\delta}\left(i s_{4}-\omega\right)\right)}{2 \omega(\omega-W)(\omega+W)}\right) \\
& \times \delta\left(q_{4}-s_{4}\right) \delta^{(3)}\left(\vec{q}-\vec{p}-\vec{p}^{\prime}\right) .
\end{aligned}
$$

Now, evaluating conveniently the delta functions, we write

$$
\begin{aligned}
\operatorname{Disc}\left[\mathcal{M}\left(s_{E}\right)\right]= & -e^{2} V^{\mu} V^{* \nu} \times \int \frac{d^{4} q_{E}}{(2 \pi)^{4}} \sum_{\lambda}\left(\varepsilon_{E \mu}^{(\lambda)} \varepsilon_{E \nu}^{*(\lambda)}\right)_{q_{4}=s_{4}}(2 \pi) \delta\left(q_{4}-s_{4}\right) \delta^{(3)}\left(\vec{q}-\vec{p}-\vec{p}^{\prime}\right) \\
& \times\left[\frac{\tilde{\delta}\left(i q_{4}+\omega\right)\left(-1+i \lambda \beta \sqrt{\gamma q_{E}^{2}}\right)_{q_{4}=i \omega}}{\beta^{2} \gamma\left(q_{4}+i \omega\right)\left(q_{4}-i W\right)\left(q_{4}+i W\right)}+\frac{\tilde{\delta}\left(i q_{4}-\omega\right)\left(-1+i \lambda \beta \sqrt{\gamma q_{E}^{2}}\right)_{q_{4}=-i \omega}}{\beta^{2} \gamma\left(-q_{4}+i \omega\right)\left(q_{4}-i W\right)\left(q_{4}+i W\right)}\right] .
\end{aligned}
$$

We can obtain this expression in an equivalent way by introducing a physical delta function $\tilde{\delta}$ allowing to select only asymptotic degrees of freedom in Hilbert space. In Ref. [28], such delta function has been used to test unitarity in a higherorder Lorentz-violating scalar theory.

The square brackets above can be written as

$$
\begin{aligned}
{\left[\theta\left(i s_{4}\right)+\theta\left(-i s_{4}\right)\right] \bar{\delta}\left(-q_{E}^{2}-a_{E} \sqrt{D_{E}}\right)=} & \frac{\theta\left(i s_{4}\right)\left(-1+i \lambda \beta \sqrt{\gamma q_{E}^{2}}\right)_{q_{4}=i \omega} \tilde{\delta}\left(i q_{4}+\omega\right)}{\beta^{2} \gamma\left(q_{4}+i \omega\right)\left(q_{4}-i W\right)\left(q_{4}+i W\right)} \\
& +\frac{\theta\left(-i s_{4}\right)\left(-1+i \lambda \beta \sqrt{\gamma q_{E}^{2}}\right)_{q_{4}=-i \omega} \tilde{\delta}\left(i q_{4}-\omega\right)}{\beta^{2} \gamma\left(-q_{4}+i \omega\right)\left(q_{4}-i W\right)\left(q_{4}+i W\right)}
\end{aligned}
$$

where one has to restrict to purely imaginary values of $s_{4}$, which is precisely the case we seek to perform the inverse transformation of time variable. This allows us to write

$\operatorname{Disc}\left[\mathcal{M}\left(s_{E}\right)\right]=-e^{2} \int \frac{d^{4} q_{E}}{(2 \pi)^{4}} \sum_{\lambda}\left(V^{\mu} \varepsilon_{E \mu}^{(\lambda)}\right)\left(V^{* \nu} \varepsilon_{E \nu}^{*(\lambda)}\right)\left[\theta\left(i s_{4}\right)+\theta\left(-i s_{4}\right)\right](2 \pi) \bar{\delta}\left(-q_{E}^{2}-a_{E} \sqrt{D_{E}}\right) \delta^{(4)}\left(q_{E}-p_{E}-p_{E}^{\prime}\right)$.

Now, we integrate and consider the inverse transformation of external momenta in terms of $s_{0}$ and use $\operatorname{Disc}[\mathcal{M}]=2 i \operatorname{Im} \mathcal{M}$, to arrive at 


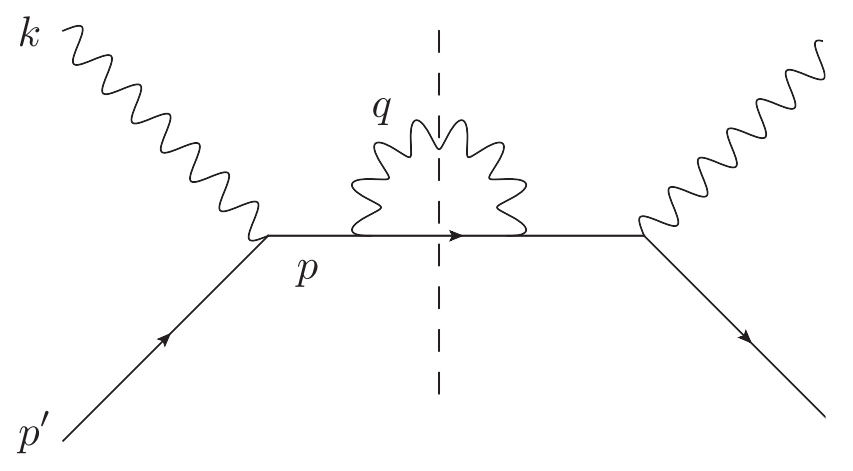

FIG. 6. The Compton scattering process at one-loop level.

$$
\begin{aligned}
2 \operatorname{Im} \mathcal{M}(s)= & \int \frac{d^{4} q}{(2 \pi)^{4}} \sum_{\lambda}\left|M_{\lambda}\right|^{2}\left[\theta\left(q_{0}\right)+\theta\left(-q_{0}\right)\right] \\
& \times(2 \pi) \bar{\delta}\left(q^{2}-a \sqrt{D}\right) \delta^{(4)}\left(q-p-p^{\prime}\right),
\end{aligned}
$$

where

$$
M_{\lambda}=(-i e) V^{\mu} \varepsilon_{\mu}^{(\lambda)}
$$

We see that it is equivalent to considering the denominators on shell in the original expression or replacing the propagator with the physical delta function. Therefore, the constraint given by unitarity is satisfied.

\section{B. Compton scattering at the one-loop level}

Now, we consider the Compton scattering process at the one-loop level. It is presented by Fig. 6 . We set $u^{\mu}=$ $(1,0,0,0)$ so that no ghosts appear. In this case, the dispersion relation turns out to be

$$
\left(q^{2}\right)^{2}-\frac{16}{M^{2}}\left(q_{0}^{2}\left(c_{1}-c_{2}\right)+c_{2}|\vec{q}|^{2}\right)^{2}|\vec{q}|^{2}=0 .
$$

Solving, we find the positive solutions

$$
\omega_{\lambda^{\prime}}=\frac{|\vec{q}| \sqrt{1-\lambda^{\prime} g c_{2}|\vec{q}|}}{\sqrt{1+\lambda^{\prime} g\left(c_{1}-c_{2}\right)|\vec{q}|}}
$$

where $g=4 / M$ and $\lambda^{\prime}= \pm 1$.

The scattering amplitude, with the help of the propagator (26), is found to be

$$
i \mathcal{M}=-\sum_{\lambda^{\prime}} e^{4} J^{* \mu}\left(p^{\prime}, k, p\right) \int \frac{d^{4} q}{(2 \pi)^{4}} \frac{(\not p-q q+m) \varepsilon_{\mu}\left(q, \lambda^{\prime}\right) \varepsilon_{\nu}^{*}\left(q, \lambda^{\prime}\right)}{\left((p-q)^{2}-m^{2}+i \epsilon\right)\left(q^{2}+\lambda^{\prime} g\left(q_{0}^{2}\left(c_{1}-c_{2}\right)+c_{2}|\vec{q}|^{2}\right)|\vec{q}|+i \epsilon\right)} J^{\nu}\left(p^{\prime}, k, p\right),
$$

with

$$
J^{\nu}\left(p^{\prime}, k, p\right)=\frac{1}{\left(p^{2}-m^{2}\right)} \gamma^{\nu}(\not p+m) \gamma^{\alpha} u^{s}\left(p^{\prime}\right) \varepsilon_{\alpha}(k, \lambda), \quad J^{* \mu}\left(p^{\prime}, k, p\right)=\frac{1}{\left(p^{2}-m^{2}\right)} \varepsilon_{\beta}^{*}(k, \lambda) \bar{u}^{s}\left(p^{\prime}\right) \gamma^{\beta}(\not p+m) \gamma^{\mu} .
$$

We focus on the integral

$$
I_{\mu \nu}(p)=\int \frac{d^{4} q}{(2 \pi)^{4}} \frac{(\not p-\not q+m) \varepsilon_{\mu}\left(q, \lambda^{\prime}\right) \varepsilon_{\nu}^{*}\left(q, \lambda^{\prime}\right)}{\left((p-q)^{2}-m^{2}+i \epsilon\right)\left(q^{2}+\lambda^{\prime} g\left(q_{0}^{2}\left(c_{1}-c_{2}\right)+c_{2}|\vec{q}|^{2}\right)|\vec{q}|+i \epsilon\right)} .
$$

In terms of the poles from Eq. (96) and the fermion one, $E_{q-p}=\sqrt{(\vec{q}-\vec{p})^{2}-m^{2}}$, we write

$$
I_{\mu \nu}(p)=\int \frac{d^{3} q d q_{0}}{(2 \pi)^{4}} \frac{F_{\mu \nu}(p-q, q)}{\left(q_{0}-p_{0}-E_{q-p}+i \epsilon\right)\left(q_{0}-p_{0}+E_{q-p}-i \epsilon\right)} \frac{1}{\left(1+\lambda^{\prime} g\left(c_{1}-c_{2}\right)|\vec{q}|\right)\left(q_{0}-\omega_{\lambda^{\prime}}+i \epsilon\right)\left(q_{0}+\omega_{\lambda^{\prime}}-i \epsilon\right)},
$$

where

$$
F_{\mu \nu}(p-q, q)=(\not p-q+m) \varepsilon_{\mu}\left(q, \lambda^{\prime}\right) \varepsilon_{\nu}^{*}\left(q, \lambda^{\prime}\right) .
$$

We perform the $q_{0}$ integral by closing the contour downward and using the residue theorem. Taking into account the relevant poles in the fourth quadrant, we arrive at

$$
\begin{aligned}
I_{\mu \nu}(p)= & \int \frac{d^{3} q}{(2 \pi)^{4}} \frac{(-2 \pi i)}{\left(1+\lambda^{\prime} g\left(c_{1}-c_{2}\right)|\vec{q}|\right)}\left[\frac{\left[F_{\mu \nu}(p-q, q)\right]_{q_{0}=p_{0}+E_{q-p}-i \epsilon}}{2 E_{q-p}\left(E_{q-p}+p_{0}-\omega_{\lambda^{\prime}}\right)\left(E_{q-p}+p_{0}+\omega_{\lambda^{\prime}}-i \epsilon\right)}\right. \\
& \left.-\frac{\left[F_{\mu \nu}(p-q, q)\right]_{q_{0}=\omega_{\lambda^{\prime}}-i \epsilon}}{2 \omega_{\lambda^{\prime}}\left(E_{q-p}+p_{0}-\omega_{\lambda^{\prime}}\right)\left(E_{q-p}-p_{0}+\omega_{\lambda^{\prime}}-i \epsilon\right)}\right] .
\end{aligned}
$$


Using Eq. (86), the discontinuity of the integral turns out to be equal to

$$
\operatorname{Disc}[\mathcal{M}(p)]=i \sum_{\lambda^{\prime}} J^{\mu}\left(p^{\prime}, k, p\right) Q_{\mu \nu}(p) J^{\nu}\left(p^{\prime}, k, p\right),
$$

where $Q_{\mu \nu}(p)=\operatorname{Disc}\left[I_{\mu \nu}(p)\right]$, such that

$$
\begin{aligned}
Q_{\mu \nu}(p)= & -\int \frac{d^{3} q}{(2 \pi)^{4}} \frac{(2 \pi)^{2}}{\left(1+\lambda^{\prime} g\left(c_{1}-c_{2}\right)|\vec{q}|\right)}\left[\frac{\left[F_{\mu \nu}(p-q, q)\right]_{q_{0}=p_{0}+E_{q-p}} \delta\left(E_{q-p}+\omega_{\lambda^{\prime}}+p_{0}\right)}{2 E_{q-p}\left(E_{q-p}+p_{0}-\omega_{\lambda^{\prime}}\right)}\right. \\
& \left.-\frac{\left[F_{\mu \nu}(p-q, q)\right]_{q_{0}=\omega_{\lambda^{\prime}}} \delta\left(E_{q-p}+\omega_{\lambda^{\prime}}-p_{0}\right)}{2 \omega_{\lambda^{\prime}}\left(E_{q-p}+p_{0}-\omega_{\lambda^{\prime}}\right)}\right] .
\end{aligned}
$$

We have set $\epsilon=0$ in the numerators, where the $\epsilon$ factors are not relevant. Using the delta function, we can simplify the denominators more, i.e.

$$
\begin{aligned}
Q_{\mu \nu}(p)= & \int \frac{d^{3} q}{(2 \pi)^{4}} \frac{(2 \pi)^{2}}{\left(1+\lambda^{\prime} g\left(c_{1}-c_{2}\right)|\vec{q}|\right)}\left[\frac{\left[F_{\mu \nu}(p-q, q)\right]_{q_{0}=p_{0}+E_{q-p}} \delta\left(E_{q-p}+\omega_{\lambda^{\prime}}+p_{0}\right)}{\left(2 E_{q-p}\right)\left(2 \omega_{\lambda^{\prime}}\right)}\right. \\
& \left.+\frac{\left[F_{\mu \nu}(p-q, q)\right]_{q_{0}=\omega_{\lambda^{\prime}}} \delta\left(E_{q-p}+\omega_{\lambda^{\prime}}-p_{0}\right)}{\left(2 E_{q-p}\right)\left(2 \omega_{\lambda^{\prime}}\right)}\right] .
\end{aligned}
$$

With the help of the identity $\int d^{3} q=\int d^{3} k d^{3} k^{\prime} \delta^{(3)}\left(\vec{k}+\vec{k}^{\prime}-\vec{p}\right)$, and introducing two additional integrals in $k_{0}$ and $k_{0}^{\prime}$ and with $k^{\prime}=p-q, k=q$, we can write

$$
\begin{aligned}
Q_{\mu \nu}(p)= & \int \frac{d^{4} k d^{4} k^{\prime}}{(2 \pi)^{4}} \frac{(2 \pi)^{2}}{\left(1+\lambda^{\prime} g\left(c_{1}-c_{2}\right)|\vec{k}|\right)}\left[\frac{\left[F_{\mu \nu}(p-q, q)\right]_{q_{0}=p_{0}-k_{0}^{\prime}} \delta\left(k_{0}+k_{0}^{\prime}-p_{0}\right) \delta\left(k_{0}+\omega_{\lambda^{\prime}}(k)\right) \delta\left(k_{0}^{\prime}+E_{k^{\prime}}\right)}{\left(2 E_{k^{\prime}}\right)\left(2 \omega_{\lambda^{\prime}}(k)\right)}\right. \\
& \left.+\frac{\left[F_{\mu \nu}(p-q, q)\right]_{q_{0}=k_{0}} \delta\left(k_{0}+k_{0}^{\prime}-p_{0}\right) \delta\left(k_{0}-\omega_{\lambda^{\prime}}(k)\right) \delta\left(k_{0}^{\prime}-E_{k^{\prime}}\right)}{\left(2 E_{k^{\prime}}\right)\left(2 \omega_{\lambda^{\prime}}(k)\right)}\right] \delta^{(3)}\left(\vec{k}+\vec{k}^{\prime}-\vec{p}\right) .
\end{aligned}
$$

Now, we use the fact that under the integral with the delta functions the $F_{\mu \nu}(p-q, q)$ factors behave as

$$
\left[F_{\mu \nu}(p-q, q)\right]_{q_{0}=p_{0}-k_{0}^{\prime}}=\left[F_{\mu \nu}(p-q, q)\right]_{q_{0}=k_{0}}=\left(\not k^{\prime}+m\right) \varepsilon_{\mu}\left(k, \lambda^{\prime}\right) \varepsilon_{\nu}^{*}\left(k, \lambda^{\prime}\right),
$$

and together with the on-shell relation

$$
\left(\not k^{\prime}+m\right)=\sum_{s^{\prime}} u^{s^{\prime}}\left(k^{\prime}\right) \bar{u}^{s^{\prime}}\left(k^{\prime}\right),
$$

we can rewrite (106) as

$$
\begin{aligned}
Q_{\mu \nu}(p)= & \sum_{s^{\prime}} \int \frac{d^{4} k}{(2 \pi)^{4}} \frac{d^{4} k^{\prime}}{(2 \pi)^{4}} u^{s^{\prime}}\left(k^{\prime}\right) \bar{u}^{s^{\prime}}\left(k^{\prime}\right) \varepsilon_{\mu}\left(k, \lambda^{\prime}\right) \varepsilon_{\nu}^{*}\left(k, \lambda^{\prime}\right)(2 \pi) \delta\left(k^{2}+\lambda^{\prime} g\left(k_{0}^{2}\left(c_{1}-c_{2}\right)+c_{2}|\vec{k}|^{2}\right)|\vec{k}|\right) \\
& \times(2 \pi) \delta\left(k^{\prime 2}-m^{2}\right)\left[\theta\left(k_{0}\right) \theta\left(k_{0}^{\prime}\right)+\theta\left(-k_{0}\right) \theta\left(-k_{0}^{\prime}\right)\right](2 \pi)^{4} \delta^{(4)}\left(k+k^{\prime}-p\right) .
\end{aligned}
$$

Considering $\operatorname{Disc}[\mathcal{M}(p)]=2 i \operatorname{Im}[\mathcal{M}(p)]$, finally, one has

$$
\begin{aligned}
2 \operatorname{Im}[\mathcal{M}(p)]= & \sum_{\lambda^{\prime}, s^{\prime}} \int \frac{d^{4} k}{(2 \pi)^{4}} \frac{d^{4} k^{\prime}}{(2 \pi)^{4}}|\tilde{M}|^{2}\left(\theta\left(k_{0}\right) \theta\left(k_{0}^{\prime}\right)+\theta\left(-k_{0}\right) \theta\left(-k_{0}^{\prime}\right)\right) \\
& \times(2 \pi)^{4} \delta^{(4)}\left(k+k^{\prime}-p\right)(2 \pi) \delta\left(k^{2}+\lambda^{\prime} g\left(k_{0}^{2}\left(c_{1}-c_{2}\right)+c_{2}|\vec{k}|^{2}\right)|\vec{k}|\right)(2 \pi) \delta\left(k^{\prime 2}-m^{2}\right),
\end{aligned}
$$

where $\tilde{M}$ is the diagram obtained by replacing the propagators by delta functions after the cutting, i.e., 


$$
\begin{aligned}
\tilde{M}= & -i e^{2} \frac{1}{\left(p^{2}-m^{2}\right)} \varepsilon_{\alpha}^{*}\left(k, \lambda^{\prime}\right) \bar{u}^{s^{\prime}}\left(p^{\prime}\right) \gamma^{\alpha}(\not p+m) \\
& \times \gamma^{\beta} u^{s}\left(p^{\prime}\right) \varepsilon_{\beta}(k, \lambda) .
\end{aligned}
$$

Hence, we conclude that the optical theorem is satisfied both at the tree level and the one-loop level within this scattering process. Since it is natural to expect that the higher-loop situation does not differ too much, we conclude that unitarity is maintained in our theory.

\section{SUMMARY}

We considered the higher-derivative Lorentz-breaking extension of QED, which involves, first, additive terms, that is, Myers-Pospelov and higher-derivative CFJ-like terms, in the purely gauge sector and, second, a new, nonrenormalizable spinor-vector coupling. For this model, we discussed the dispersion relations and found that, to achieve tree-level unitarity, either only one higherderivative term, that is, the MP term or the higher-derivative CFJ term, can be present in the action, or the Lorentzbreaking vector must be not simply timelike but directed along the time axis. Apart from this, we carried out a study of quantum corrections to two-point functions of gauge and spinor fields and showed that for a consistent subtraction of the divergences the corresponding higher-derivative terms should be introduced from the very beginning, both in gauge and spinor sectors, with the structure of quantum corrections is simplified for the lightlike Lorentz-breaking vectors. Nevertheless, it is very reasonable to treat this theory as an effective one, aimed for studying of the lowenergy domain. Indeed, all higher-order divergent terms will be very small since they are proportional to different degrees of $\frac{1}{M}$, with $M$ assumed to be of the order of the Planck mass; thus, they are strongly suppressed. One can argue that a similar situation will occur in higher loops where all dangerous divergences will be suppressed by negative degrees of $M$. We carried out a calculation of these corrections in the finite-temperature case as well, and we see that our result tends to zero in the high-temperature limit.

We verified unitarity in our theory, both at the tree level and at the one-loop level. We checked directly that the optical theorem is satisfied in both cases; therefore, we conclude that, even in the presence of higher time derivatives, unitarity in our theory is preserved for the processes we have considered, which raises the hope that other situations and, in particular, other field theory models, where higher time derivatives do not jeopardize unitarity, are also possible. We conclude that this manner of introducing the higher derivatives is compatible with unitarity as well as the Horava-Lifshitz methodology in which only higher spatial derivatives are present. However, the advantage of our approach is that, unlike the HoravaLifshitz theories [29], in our case, the Lorentz symmetry breaking continues to be small, which is much more reasonable from the viewpoint of achieving the consistency with experimental measurements, which, as is well known [2], impose very strong upper boundaries on Lorentzbreaking effects.

\section{ACKNOWLEDGMENTS}

This work was partially supported by Conselho Nacional de Desenvolvimento Científico e Tecnológico (CNPq). The work by A. Yu. P. has been supported by the CNPq Project No. 303783/2015-0.
[1] D. Colladay and V. A. Kostelecky, Phys. Rev. D 55, 6760 (1997); 58, 116002 (1998).

[2] V. A. Kostelecky and N. Russell, Rev. Mod. Phys. 83, 11 (2011).

[3] D. Fliegner, M. G. Schmidt, and C. Schubert, Z. Phys. C 64, 111 (1994); D. Fliegner, P. Haberl, M. G. Schmidt, and C. Schubert, Ann. Phys. (N.Y.) 264, 51 (1998); A. Castro, J. L. Davis, P. Kraus, and F. Arsen, J. High Energy Phys. 06 (2007) 007.

[4] R. C. Myers and M. Pospelov, Phys. Rev. Lett. 90, 211601 (2003).

[5] C. M. Reyes, Phys. Rev. D 82, 125036 (2010); 87, 125028 (2013).

[6] O. Ganguly, D. Gangopadhyay, and P. Majumdar, Europhys. Lett. 96, 61001 (2011).

[7] P. A. Bolokhov and M. Pospelov, Phys. Rev. D 77, 025022 (2008).
[8] T. Mariz, Phys. Rev. D 83, 045018 (2011).

[9] V. A. Kostelecky and M. Mewes, Phys. Rev. D 80, 015020 (2009).

[10] J. Leite, T. Mariz, and W. Serafim, J. Phys. G 40, 075003 (2013).

[11] T. Mariz, J. R. Nascimento, and A. Yu. Petrov, Phys. Rev. D 85, 125003 (2012).

[12] V. A. Kostelecky and M. Mewes, Phys. Rev. Lett. 99, 011601 (2007).

[13] L. Campanelli, Phys. Rev. D 90, 105014 (2014).

[14] A. Kostelecky and M. Mewes, Phys. Rev. D 88, 096006 (2013).

[15] J. R. Nascimento, A. Yu. Petrov, and C. Marat Reyes, Phys. Rev. D 92, 045030 (2015).

[16] R. Casana, M. M. Ferreira, Jr., R. V. Maluf, and F. E. P. dos Santos, Phys. Lett. B 726, 815 (2013).

[17] H. Georgi, Annu. Rev. Nucl. Part. Sci. 43, 209 (1993). 
[18] I. L. Buchbinder, M. Cvetic, and A. Y. Petrov, Nucl. Phys. B571, 358 (2000).

[19] A. Celeste, T. Mariz, J. R. Nascimento, and A. Yu. Petrov, Phys. Rev. D 93, 065012 (2016).

[20] T. D. Lee and G. C. Wick, Nucl. Phys. B9, 209 (1969); Phys. Rev. D 2, 1033 (1970).

[21] R. E. Cutkosky, P. V. Landshoff, D. I. Olive, and J. C. Polkinghorne, Nucl. Phys. B12, 281 (1969).

[22] S. W. Hawking and T. Hertog, Phys. Rev. D 65, 103515 (2002); I. Antoniadis, E. Dudas, and D. M. Ghilencea, Nucl. Phys. B767, 29 (2007); J. Garriga and A. Vilenkin, J. Cosmol. Astropart. Phys. 01 (2013) 036.

[23] J. Lopez-Sarrion and C. M. Reyes, Eur. Phys. J. C 73, 2391 (2013); C. M. Reyes and L. F. Urrutia, Phys. Rev. D 95, 015024 (2017).
[24] M. Maniatis and C. M. Reyes, Phys. Rev. D 89, 056009 (2014).

[25] D. Anselmi and M. Piva, J. High Energy Phys. 06 (2017) 066.

[26] D. Anselmi and M. Piva, Phys. Rev. D 96, 045009 (2017).

[27] B. Charneski, M. Gomes, R. V. Maluf, and A. J. da Silva, Phys. Rev. D 86, 045003 (2012); R. Casana, M. M. Ferreira, R. V. Maluf, and F.E. P. dos Santos, Phys. Rev. D 86, 125033 (2012).

[28] L. Balart, C. M. Reyes, S. Ossandon, and C. Reyes, Phys. Rev. D 98, 035035 (2018).

[29] P. Horava, Phys. Rev. D 79, 084008 (2009). 